\title{
L'evoluzione degli atteggiamenti verso la matematica e il suo insegnamento degli insegnanti di scuola elementare in formazione iniziale
}

\author{
The evolution of preservice primary school teachers' attitudes \\ towards mathematics and its teaching
}

\author{
Monica Panero ${ }^{\star}$, Pietro Di Martino ${ }^{\circ}$, Luciana Castelli ${ }^{\star}$ e Silvia Sbaragli ${ }^{\star}$ \\ *Dipartimento formazione e apprendimento, SUPSI, Locarno - Svizzera \\ ${ }^{\circ}$ Dipartimento di Matematica, Università di Pisa - Italia \\ 凶monica.panero@supsi.ch, pietro.di.martino@unipi.it, \\ luciana.castelli@supsi.ch, silvia.sbaragli@supsi.ch.
}

Sunto / Il percorso formativo come docenti di scuola elementare in didattica della matematica può essere fortemente influenzato da fattori di natura affettiva, che, a loro volta, risultano spesso legati ad esperienze scolastiche negative vissute con la matematica. Il progetto di ricerca-azione qui descritto ha approfondito proprio questo fenomeno, focalizzandosi sui futuri docenti di scuola elementare del Canton Ticino, con un duplice obiettivo: da un lato, progettare e implementare efficaci pratiche formative per lo sviluppo di atteggiamenti positivi verso la matematica e il suo insegnamento; dall'altro, studiare l'evoluzione di tali atteggiamenti nell'arco dei primi due anni della formazione. Sono stati sviluppati specifici interventi didattici e strumenti di osservazione che hanno permesso di rilevare e monitorare gli atteggiamenti degli studenti e di analizzare quali dimensioni - disposizione emozionale, senso di autoefficacia, visione della disciplina (Di Martino \& Zan, 2011) - sono state più o meno influenti sul cambiamento di atteggiamento, e su quali componenti quindi la formazione può cercare di intervenire in modo più incisivo ed efficace.

Parole chiave: atteggiamenti; emozioni; autoefficacia; formazione dei docenti; scuola elementare.
Abstract / The training as primary school teachers in mathematics education can be strongly influenced by emotional factors, which, in turn, are often linked to negative school experiences with mathematics. The research-action project described in this article has deepened on this phenomenon focusing on future primary school teachers in Canton Ticino, with a twofold objective: on the one hand, to design and implement effective training practices for the development of positive attitudes towards mathematics and its teaching; on the other hand, to study the evolution of such attitudes during the first two years of training. Specific didactic interventions and observation tools have been developed to detect and monitor students' attitudes and to analyse which dimensions - emotional disposition, sense of self-efficacy, vision of the discipline (Di Martino \& Zan, 2011) - have been more or less influential on the change of attitude and on which components therefore training can try to intervene more incisively and effectively.

Keywords: attitudes; emotions; self-efficacy; teacher training; primary school. 


\section{Introduzione}

Il progetto di ricerca-azione "Evoluzione degli atteggiamenti verso la matematica e il suo insegnamento" (AMI) ${ }^{1}$ della durata di tre anni (settembre 2017 - agosto 2020) si è focalizzato sull'analisi dell'evoluzione degli atteggiamenti verso la matematica e il suo insegnamento da parte dei futuri insegnanti di scuola elementare, anche a seguito di interventi formativi progettati e proposti appositamente per sviluppare o rafforzare un atteggiamento positivo. II progetto ha coinvolto la coorte di studenti immatricolati nell'a.a. 2017/18 al Bachelor in "Insegnamento per il livello elementare" offerto dal Dipartimento formazione e apprendimento della Scuola universitaria professionale della Svizzera italiana (SUPSI) di Locarno, attraverso un disegno di ricerca longitudinale condotta sui primi due anni di formazione. II percorso di formazione seguito dagli studenti ha una durata complessiva di tre anni e permette di diventare docenti generalisti nelle scuole elementari del Canton Ticino. Una parte importante e caratterizzante della formazione è la pratica professionale realizzata nelle scuole, dove gli studenti osservano, progettano e realizzano in prima persona percorsi didattici. Sui moduli professionali, in cui gli studenti vengono accompagnati e guidati nella riflessione sulle proprie pratiche e sul proprio sviluppo personale e identitario come futuri docenti, si innestano i moduli di matematica, per orientare le progettazioni e le riflessioni dei futuri docenti verso questioni di natura disciplinare e didattica.

Nel contesto della formazione dei docenti di scuola elementare, come riscontrato in diverse ricerche a livello internazionale (si vedano ad esempio Bursal \& Paznokas, 2006; Coppola, Di Martino, Pacelli \& Sabena, 2012; Coppola, Di Martino, Mollo, Pacelli \& Sabena, 2013; Di Martino \& Sabena, 2011; Hannula, Liljedahl, Kaasila \& Rösken, 2007; Wood, 1987), molti studenti, pur provenendo da percorsi scolastici diversi tra loro, hanno avuto un'esperienza piuttosto negativa con la matematica, sviluppando un atteggiamento generalmente avverso alla disciplina che può rappresentare un ostacolo al loro percorso formativo come futuri docenti. La ricerca in tal senso evidenzia infatti come un atteggiamento di questo tipo possa «seriamente interferire con il fatto che gli studenti diventino bravi insegnanti di matematica» (Hannula et al., 2007, p. 153, traduzione degli autori), incidendo sia nel percorso formativo per diventare insegnanti, sia nelle loro future scelte didattiche.

Già nel 1978, Mihalko sottolineava l'importanza di rompere il ciclo della "mathophobia" (la fobia della matematica) riconoscendo nella formazione dei futuri docenti il momento cruciale per intervenire: «Non ci si può aspettare che [gli insegnanti di matematica] generino entusiasmo ed eccitazione per una materia per la quale provano paura e ansia. Se vogliamo spezzare il ciclo della fobia della matematica, questo deve accadere nella formazione degli insegnanti» (Mihalko, 1978, p. 36, traduzione degli autori).

La ricerca in didattica della matematica ha iniziato a sviluppare un interesse specifico per lo studio dei fattori affettivi legati alla disciplina a partire dalla metà degli anni ' 80 , con la nascita di un filone di studi focalizzato inizialmente sulle convinzioni (beliefs) degli studenti, e nato con l'obiettivo di interpretare, all'interno di attività di problem solving, fenomeni difficili da spiegare in termini puramente cognitivi (Schoenfeld, 1983). In particolare, Schoenfeld (1992) ha messo in risalto come gli studenti vedano e concettualizzino in modo diverso la matematica e come la loro visione della disciplina influenzi il loro comportamento e la loro presa di decisioni mentre affrontano e risolvono un problema matematico. Nello stesso periodo, basandosi sugli studi di Thompson (1992), I'interesse di questo filone di ricerca si è spostato anche sulle convinzioni degli insegnanti riguardo la matematica, mettendo in evidenza come la visione della matematica di un docente influenzi le sue scelte didattiche (Calderhead, 1996; Hodgen \& Askew, 2011; Sbaragli, 2006) nonché le interazioni con gli studenti (Buehl, Alexander \& Murphy, 2002). Questi studi, incentrati sulle convinzioni di studenti e insegnanti

1. Oltre agli autori di questo articolo, hanno collaborato al progetto Francesca Antonini, responsabile del Bachelor in "Insegnamento per il livello elementare", e Alberto Piatti, direttore del Dipartimento. 
riguardo la matematica, sono stati il primo passo per andare oltre a un approccio puramente cognitivo in didattica della matematica, prendendo in considerazione l'influenza di fattori affettivi, quali emozioni, valori, atteggiamenti, oltre a credenze e convinzioni, nel processo di insegnamentoapprendimento (McLeod, 1992).

Questo filone di ricerca ha aperto un campo completamente inesplorato: fino a quel momento infatti le ricerche condotte nel contesto della formazione degli insegnanti si erano focalizzate sul determinare quali saperi disciplinari, didattici e pedagogici, fossero necessari per insegnare la matematica a diversi livelli scolastici, con risultati sicuramente molto interessanti prodotti nel filone di quella che viene chiamata la Mathematical Knowledge for Teaching (si veda per esempio Hill et al., 2008). D'altra parte, l'esclusione della sfera affettiva dalle ricerche sullo sviluppo professionale dei docenti (in formazione e in servizio) ha comportato nel tempo importanti lacune nell'interpretazione di fenomeni legati all'insegnamento e all'apprendimento. È ormai infatti assodato che ogni individuo si approccia ai saperi da acquisire e da insegnare in modi personali fortemente dipendenti da aspetti affettivi, quali le emozioni che ha provato e prova nei confronti della disciplina nella sua esperienza come studente, le competenze che crede di possedere, le convinzioni sui contenuti disciplinari da apprendere o da spiegare agli altri e la propria identità come insegnante di matematica.

Nel caso specifico degli insegnanti di scuola elementare, come già in parte accennato, tali esperienze, emozioni e atteggiamenti nei confronti della disciplina hanno spesso una connotazione negativa. Risulta quindi cruciale che un percorso di formazione per futuri docenti di scuola elementare crei le condizioni per rafforzare e sviluppare un atteggiamento positivo verso la matematica e verso il suo insegnamento.

\section{Le tre dimensioni dell'atteggiamento}

Nel campo della ricerca in didattica della matematica è stato elaborato un modello teorico ormai consolidato che descrive l'atteggiamento nei confronti della matematica come un costrutto tridimensionale (Di Martino \& Zan, 2011) composto da emozioni associate alla matematica, convinzioni su sé stessi in relazione alla matematica e convinzioni sulla matematica. L'atteggiamento verso la matematica viene dunque descritto e analizzato considerando tre componenti e le loro mutue relazioni: la disposizione emozionale, il senso di autoefficacia, la visione della disciplina.

La disposizione emozionale verso la disciplina si manifesta tipicamente con espressioni come «Mi piace/non mi piace la matematica perché...»; la percezione di riuscire o non riuscire in matematica, ovvero il senso di autoefficacia individuato in letteratura come "competenza percepita" (Pajares \& Miller, 1994), si trova espressa in forme linguistiche del tipo «Riesco/non riesco...»; infine, la visione della matematica è tipicamente introdotta da frasi che cominciano nel seguente modo: "La matematica è...».

Le analisi condotte con il modello tridimensionale dell'atteggiamento (Di Martino \& Zan, 2011) hanno mostrato come le tre componenti siano profondamente interconnesse. Sono emerse, in particolare, interessanti relazioni tra disposizioni emotive negative verso la matematica e le altre componenti dell'atteggiamento. Nello specifico, gli studi condotti da Di Martino e Zan hanno mostrato come una disposizione emozionale negativa verso la matematica possa essere dovuta a diversi fattori legati al senso di autoefficacia e alla visione della matematica. Inoltre, il senso di autoefficacia in matematica è strettamente legato alla visione che lo studente ha del successo e dell'errore in matematica ( $\mathrm{Di}$ Martino \& Zan, 2011).

Nei prossimi paragrafi (par. 2.1, 2.2, 2.3) approfondiamo ciascuna delle tre dimensioni del costrutto di atteggiamento verso la matematica preso in considerazione. 


\subsection{Disposizione emozionale}

In ambito educativo, Pekrun e il suo gruppo di ricerca hanno condotto interessanti studi sulle emozioni legate all'apprendimento (Pekrun, 2006; Pekrun, Frenzel, Goetz \& Perry, 2007; Pekrun, Vogl, Muis \& Sinatra, 2017) considerandole come «processi multidimensionali e coordinati di sottosistemi psicologici, compresi i processi fisiologici affettivi, cognitivi, motivazionali, espressivi e periferici» (Pekrun, 2006, p. 316, traduzione degli autori). II modello teorico proposto da Pekrun e colleghi risulta sufficientemente flessibile per descrivere e classificare le emozioni associate all'apprendimento, sia relative a situazioni e attività che gli studenti hanno vissuto o possono immaginare di affrontare (emozioni di riuscita legate all'attività ed emozioni epistemiche innescate dalle caratteristiche cognitive del compito), sia relative a risultati ottenuti (emozioni di riuscita legate ai risultati).

A partire da questi studi, e in linea con altre ricerche condotte nello stesso campo (si vedano ad esempio: Raccanello \& Brondino, 2017; Russel, 1980), le emozioni possono essere categorizzate come in Tabella 1, distinguendo tra:

- positive, se sono piacevoli, e negative, se sono spiacevoli;

- attivanti o deattivanti, a seconda che attivino o meno il soggetto dal punto di vista fisiologico con reazioni quali arrossamento, sudorazione, aumento del battito cardiaco ecc.

\begin{tabular}{|l|l|l|}
\hline \multirow{2}{*}{ Positive } & Attivanti & Deattivanti \\
& Piacere & Sollievo \\
& Speranza & Orgoglio \\
& Gratitudine \\
& Sorpresa (in senso positivo) \\
& Curiosità & Appagamento \\
& Rabbia & \\
\hline Negative & Ansia & \\
& Vergogna & Sconforto \\
& Frustrazione & Noia \\
& Tristezza \\
Delusione \\
Paura \\
Disgusto \\
Confusione & \\
& Affaticamento \\
\hline
\end{tabular}

Tabella 1. Categorizzazione delle emozioni legate all'apprendimento.

Usando tali categorizzazioni, Pekrun e colleghi osservano che emozioni positive attivanti nei confronti di un'attività o di un risultato (ad esempio, piacere, speranza e orgoglio) hanno influenze positive sulla motivazione dello studente, lo rendono flessibile nella scelta delle strategie di apprendimento e supportano la sua capacità di autoregolazione, influenzando positivamente nella maggior parte dei casi la sua performance accademica. Al contrario, le emozioni negative deattivanti, come sconforto e noia, possono ridurre la motivazione e la partecipazione dello studente, avendo conseguenze negative anche sulla sua performance (Pekrun, 2006). Per quanto riguarda le emozioni positive deattivanti o quelle negative attivanti, gli effetti sono più complessi da essere interpretati; ciò comporta che tali emozioni possano favorire o al contrario ostacolare l'apprendimento degli allievi (Rowe \& Fitness, 2018).

Che le emozioni influenzino l'apprendimento e possano incidere sulle singole prestazioni matematiche è ormai uno dei cosiddetti "solid findings" della ricerca educativa, in particolare di quella matematica (si vedano ad esempio Antognazza, Di Martino, Pellandini \& Sbaragli, 2016; Evans, 2000; Hannula, 2002; Op 'T Eynde, De Corte \& Verschaffel, 2006).

Per quanto concerne i futuri docenti di scuola elementare, molte ricerche si sono concentrate sulle emozioni negative legate alla matematica, e in particolare sull'ansia, proponendo degli approcci efficaci per ridurre la cosiddetta "ansia da matematica" negli studenti, permettendo così di (ri)costruire un rapporto positivo con la disciplina che saranno chiamati a insegnare (Hannula et al., 2007). Questi 
approcci si basano sull'idea di far vivere ai futuri docenti delle esperienze positive con la matematica, proponendo attività manipolative per impararla in modo costruttivo e attività sfidanti di problem solving e di scoperta di congetture e proprietà. II tutto in un clima d'aula che consacra uno spazio importante alla discussione e alle domande, promuove la riflessione e non stigmatizza l'errore. Tali metodologie possono aiutare i formatori a «fornire loro [agli studenti] I'opportunità di cambiare. [...] possiamo parlare di dare possibilità agli studenti, o di creare occasioni favorevoli al cambiamento in coloro che soffrono di ansia da matematica» (Hannula et al., 2007, p. 156, traduzione degli autori). Altri studi dimostrano che paura e ansia sono solo alcune delle emozioni negative provate dagli studenti e che lo spettro di emozioni da rilevare, analizzare e su cui intervenire è molto più ampio. Di Martino e Sabena (2011), in particolare, sottolineano l'importanza di analizzare in modo distinto le emozioni verso la matematica e quelle verso il suo insegnamento come «due facce diverse della stessa medaglia» (Di Martino \& Sabena, 2011, p. 91). Tra i risultati di tale ricerca, emerge che le emozioni negative verso l'insegnamento della matematica sono spesso legate al senso di autoefficacia come docente e che questa relazione è da studiare più nel dettaglio.

È stato inoltre evidenziato un fenomeno interessante tra i futuri docenti di scuola elementare che dichiarano un'emozione o un rapporto negativo con la matematica: il desiderio di una math-redemption (Coppola et al., 2013), ovvero un senso di riscatto e di rivincita sulle esperienze negative vissute con la matematica nel loro passato come allievi, una volontà di riappacificarsi con la materia, spinti dalla possibilità di insegnarla in modo diverso da quello subito. Nella formazione dei docenti, individuare, suscitare e supportare questo desiderio appare fondamentale per quegli studenti che hanno maturato una disposizione emozionale negativa nei confronti della disciplina.

\subsection{Autoefficacia}

Bandura (1997) definisce l'autoefficacia come l'insieme delle convinzioni o percezioni che un individuo ha delle proprie abilità. Nel quadro della sua teoria sociale cognitiva, viene ipotizzato che un individuo sviluppi e maturi le convinzioni sulla propria autoefficacia basandosi su quattro fonti:

- Mastery experience, ossia l'interpretazione personale dei propri risultati raggiunti; I'individuo interpreta e valuta i risultati ottenuti, crea e rivisita, in base a tali interpretazioni, giudizi personali sulle proprie competenze acquisite.

- Vicarious experience, ossia la misura delle proprie capacità accademiche, grazie al confronto con le performance positive o negative di altri compagni o di adulti scelti come punti di riferimento.

- Social persuasion, data dai feedback, dai rinforzi positivi o negativi che lo studente riceve dagli altri (genitori, insegnanti, compagni).

- Emotional and physiological states, che lo studente impara a interpretare come un indicatore di competenza personale valutando le proprie prestazioni in diverse situazioni.

Usher e Pajares (2009) hanno ripreso in seguito queste quattro dimensioni, proponendo e validando una batteria di item specifici per misurare ciascuna fonte di autoefficacia nel contesto dell'apprendimento della matematica a livello di scuola media. Tale studio dimostra che le fonti di autoefficacia individuate hanno un impatto significativo anche sulla motivazione e sulla riuscita degli studenti in matematica. Risulta inoltre confermato, come aveva già ipotizzato Bandura (1997), che la mastery experience è la più influente tra le fonti di autoefficacia.

D'altra parte, per il contesto di ricerca e intervento del progetto AMI, oltre al senso di autoefficacia in matematica, è interessante monitorare il senso di autoefficacia dei futuri insegnanti di scuola elementare come insegnanti di matematica, ovvero le convinzioni che i futuri docenti possiedono relativamente alle loro abilità e possibilità di insegnare la matematica in modo efficace. L'autoefficacia nell'insegnamento della matematica è sicuramente correlata con l'autoefficacia in matematica (Esterly, 2003; Zuya, Kwalat \& Attah, 2016), ma non è sovrapponibile ad essa: la riflessione in questo senso viene avviata e regolata costantemente dal futuro docente nel corso della propria formazione come insegnante nei corsi e seminari e grazie ai feedback che riceve dalla pratica professionale svolta in 
classe, mentre progetta, sperimenta e analizza percorsi didattici per gli allievi. È dunque evidente che i percorsi formativi forniti ai futuri docenti di scuola elementare possono incoraggiare in maniera più o meno forte le pratiche riflessive dello studente sul proprio senso di autoefficacia come insegnante di matematica.

Nell'ambito della formazione iniziale dei docenti di scuola elementare, già Kahle (2008) ha condotto una ricerca per esplorare la relazione tra autoefficacia in matematica e autoefficacia nell'insegnamento della matematica, nonché l'influenza di tali fattori sulle pratiche didattiche che i futuri docenti sperimentano in classe nel corso della loro formazione professionale. Kahle (2008) sottolinea quanto sia fondamentale che i futuri docenti abbiano sviluppato in prima persona una forte autoefficacia in matematica e nell'insegnamento della matematica, affinché possano insegnare la matematica creando connessioni tra i saperi in gioco e in modo che i loro allievi non solo imparino delle procedure ma soprattutto comprendano perché esse funzionano.

Zuya et al. (2016) hanno in seguito ripreso le teorie di Bandura (1997) e gli studi di Kahle (2008) e hanno somministrato un questionario per misurare l'autoefficacia nelle due dimensioni - in matematica e nell'insegnamento della matematica - da parte dei futuri docenti di matematica nella scuola secondaria, ottenendo risultati più alti in media per la seconda dimensione rispetto alla prima. Un risultato analogo, forse ancor più accentuato, potrebbe emergere nel caso di studenti in formazione per diventare futuri docenti di scuola elementare, che hanno dunque scelto di diventare docenti generalisti e non specializzati in matematica. Mettere alla prova gli studenti con sfide accattivanti ma sempre alla loro portata (Zuya et al., 2016), così come renderli attivi nel loro apprendimento e nella loro riflessione (Briley, 2012), sono strategie che «potrebbero portare a un cambiamento più duraturo delle loro convinzioni matematiche, dell'autoefficacia in matematica e dell'autoefficacia nell'insegnamento della matematica, che potrebbe proseguire nella loro carriera di insegnanti» (Briley, 2012, p. 10, traduzione degli autori). Tale auspicabile cambiamento, quindi, ha a che fare non solo con le convinzioni che il futuro docente matura su di sé e sul suo essere docente ma anche con le sue convinzioni sulla disciplina che andrà ad insegnare, ovvero con la sua visione della matematica, la terza e ultima dimensione che viene approfondita nel paragrafo seguente.

\subsection{Visione della matematica}

L'insieme delle convinzioni che un individuo possiede sulla matematica è, come già ricordato, uno dei primi focus di studio nell'ambito della ricerca su affect e insegnamento della matematica (Thompson, 1992). Se è vero che metodologicamente non è facile indagare quest'ambito, in quanto gli adulti tendono a non esporsi quando dichiarano le proprie convinzioni in un ambito che considerano sensibile, ed è dunque molto studiato il fenomeno della discrepanza tra convinzioni dichiarate e convinzioni in azione (Raymond, 1996), l'ipotesi alla base degli studi sulle convinzioni degli insegnanti è che la loro visione della matematica possa influenzare fortemente le loro scelte didattiche, così come l'approccio al percorso formativo degli insegnanti in ingresso. Dalla letteratura emergono convinzioni relative alla matematica con cui tipicamente gli studenti si approcciano alla formazione per diventare maestri e che spesso si ritrovano riflesse nel modo di insegnare, e che sono già visibili durante la pratica professionale o successivamente, con l'effettiva entrata in servizio. Handal (2003), nella sua revisione della letteratura, ha rilevato che gran parte degli studenti all'inizio della formazione ritengono che la matematica sia una disciplina basata su regole e procedure da memorizzare, e che in matematica esista solitamente una sola strategia o comunque una strada migliore rispetto a tutte le altre per arrivare alla soluzione, anch'essa solitamente considerata unica, in quanto in matematica ogni cosa è completamente giusta o completamente sbagliata. Altre credenze, tra le più diffuse, inoltre, vedono la matematica principalmente come una disciplina che richiede precisione e velocità, e ritengono che le abilità matematiche siano innate e che si basino sulla logica piuttosto che sull'intuizione. In relazione con queste ultime credenze, vi è la convinzione diffusa che la matematica sia una disciplina complessa e difficile da apprendere. 
Dietro ad alcune di queste convinzioni si celano visioni strumentali dell'apprendimento della matematica (Skemp, 1976), che si hanno quando la matematica viene vista come un insieme di regole, formule e procedure, e quando fare matematica significa apprenderle e utilizzarle all'occorrenza. Tale visione potrebbe influenzare pesantemente il modo in cui il futuro docente concepisce l'insegnamento della matematica, riducendolo alla semplice presentazione e ripetizione di regole ed esercizi, senza creare alcun legame tra i concetti appresi, e valutando positivamente l'apprendimento mnemonico e la riproduzione di procedure da parte degli allievi.

Al contrario, sarebbe auspicabile che una formazione indirizzata ai futuri docenti di scuola elementare possa contribuire a sviluppare una visione relazionale dell'apprendimento della matematica (Skemp, 1976), promossa dai curriculum (nello specifico, dal Piano di studio della scuola dell'obbligo ticinese: DECS, 2015), in cui ci si interroga sul perché formule e regole funzionino e si è in grado di ricostruirle anche senza ricordarle a memoria, creando e sfruttando connessioni significative tra le conoscenze apprese. Questo modo di vedere la matematica e il suo apprendimento influenza chiaramente anche il modo di insegnarla, portando a una distinzione in due grandi categorie di insegnanti in termini di visione dell'insegnamento della matematica: coloro che lo vedono come un processo cognitivo di stampo costruttivista e coloro che lo intendono come una trasmissione diretta di conoscenze (Staub \& Stern, 2002).

\section{Ipotesi e finalità del progetto}

A partire dall'assunto, già esposto nel par. 1 e confermato dalla letteratura (par. 2), secondo il quale nella maggior parte dei casi gli studenti che intraprendono un percorso di formazione per diventare docenti di scuola elementare hanno spesso un atteggiamento negativo nei confronti della matematica che si ripercuote anche sulla competenza percepita come futuri docenti di matematica, sono state identificate le seguenti ipotesi di lavoro alla base del progetto:

1. il senso di autoefficacia rispetto all'insegnamento della matematica è condizionato dall'atteggiamento nei confronti della matematica del soggetto;

2. I'atteggiamento nei confronti della matematica in tutte le sue tre componenti - emozioni, senso di autoefficacia e convinzioni - può essere modificato anche nella formazione terziaria dei futuri insegnanti attraverso interventi formativi mirati.

Il progetto AMI si è dunque sviluppato su due piani fortemente intrecciati: quello della formazione, finalizzato alla progettazione e all'implementazione di efficaci pratiche educative per lo sviluppo di un atteggiamento positivo nei confronti della matematica da parte dei futuri maestri, e quello della ricerca, finalizzato allo studio della modificabilità nel lungo periodo degli atteggiamenti e dell'efficacia di interventi multipli specificatamente sviluppati.

Questa doppia finalità ha richiesto la progettazione e lo sviluppo non solo di specifici interventi formativi, ma anche di strumenti di osservazione e di analisi per monitorare gli eventuali cambiamenti negli atteggiamenti degli studenti nel corso della formazione.

\section{Partecipanti e dispositivo di formazione}

I partecipanti allo studio sono 80 studenti iscritti nell'a.a. 2017/18 al primo anno di Bachelor in "Insegnamento per il livello elementare" (in seguito denominato "Bachelor") offerto dal Dipartimento 
formazione e apprendimento (in seguito abbreviato con DFA) della SUPSI di Locarno. Di essi, 75 risultano iscritti al primo anno per la prima volta e 5 ripetono il primo anno; inoltre, 20 sono maschi e 60 sono femmine, di età media 22 anni.

Come già anticipato nel par. 1, una parte fondamentale della formazione Bachelor è costituita dalla pratica professionale che viene svolta per i primi due anni con una frequenza di un giorno a settimana, salvo i momenti cosiddetti di "pratica blocco", che consistono in una pratica continuativa di tre settimane per ogni semestre effettuata nella classe di un "docente di pratica professionale" che accoglie lo studente e svolge il ruolo di tutor. In questi primi due anni gli studenti osservano alcune attività proposte dal docente di pratica professionale e ne progettano e realizzano altre in prima persona. Al terzo anno di formazione il tempo e l'impegno dedicati alla pratica aumentano notevolmente, poiché agli studenti è data la possibilità di assumere un incarico di docenza in classe a metà tempo (per I'altra metà del tempo sono impegnati a seguire dei corsi di approfondimento al DFA), organizzando e gestendo tutti i momenti in aula da soli con i bambini.

Per quanto riguarda la matematica, la formazione prevede 4 moduli di didattica disciplinare per i primi due anni e il seminario di Progettazione annuale di matematica al terzo anno (si veda la Tabella 2 per i dettagli). I moduli e il seminario sono progettati attorno ai tre ambiti disciplinari previsti dal Piano di studio per la scuola dell'obbligo ticinese (DECS, 2015) per quanto concerne la scuola elementare: Geometria, Grandezze e misure, Numeri e calcolo.

Ogni modulo si compone di due parti:

- Corsi. Parti teoriche che si svolgono a grande gruppo (tutti gli studenti dello stesso anno), con modalità il più possibile partecipata e dialogata. Sono basati su approfondimenti di matematica e riflessioni in ambito didattico al fine di riprendere e approfondire lo studio dei saperi di base epistemologici, disciplinari e di didattica della disciplina.

- Seminari. Parte applicativa incentrata sulla trasposizione didattica degli elementi teorici dei corsi e sull'uso e l'analisi di strumenti di progettazione per attività e percorsi matematici destinati alla classe. I seminari vengono svolti in piccoli gruppi di 25 studenti circa, in stretto legame con la loro pratica professionale.

\begin{tabular}{|l|l|}
\hline Periodo & Moduli inerenti I'area matematica \\
\hline $\begin{array}{l}\text { I anno, } \\
\text { I semestre }\end{array}$ & $\begin{array}{l}\text { Matematica I: fondamenti di didattica della Geometria (4 ECTS) } \\
- \text { Corso Geometria (24h) } \\
- \text { Seminario Geometria (24h) }\end{array}$ \\
\hline $\begin{array}{l}\text { I anno, } \\
\text { II semestre }\end{array}$ & $\begin{array}{l}\text { Matematica II: fondamenti di didattica di Grandezze e misure (4 ECTS) } \\
- \text { Corso Grandezze e misure (24h) } \\
- \text { Seminario Grandezze e misure (24h) }\end{array}$ \\
\hline II anno, & $\begin{array}{l}\text { Matematica III: la didattica nel I ciclo (4,5 ECTS) } \\
- \text { Corso Risoluzione di problemi (12h) } \\
- \text { Semestre } \\
- \text { Seminario Numeri e calcolo / ciclo (24h) }\end{array}$ \\
\hline II anno, \\
II semestre & $\begin{array}{l}\text { Matematica IV: la didattica nel II ciclo (4,5 ECTS) } \\
- \text { Corso Aspetti dell'apprendimento della matematica nel II ciclo (12h) } \\
- \text { Corso Numeri e calcolo II ciclo (24h) } \\
- \text { Seminario Numeri e calcolo II ciclo (24h) }\end{array}$ \\
\hline III anno, & $\begin{array}{l}\text { La progettazione annuale (6 ECTS), comprende tre seminari: } \\
- \text { La progettazione annuale di italiano (24h) } \\
- \text { La progettazione annuale di matematica (24h) } \\
- \text { La progettazione annuale di ambiente (24h) }\end{array}$ \\
\hline
\end{tabular}

Tabella 2. Presentazione dei moduli inerenti la didattica della matematica offerti dal Bachelor. 


\section{Procedura}

Per rilevare e monitorare l'evoluzione degli atteggiamenti degli studenti verso la matematica e verso il suo insegnamento è stato predisposto un disegno di ricerca longitudinale che ha previsto tre momenti di raccolta dei dati (Tabella 3) distribuiti su due anni, a partire da settembre 2017, in parallelo ai momenti di formazione.

La raccolta dei dati per rilevare e monitorare l'evoluzione degli atteggiamenti degli studenti è avvenuta tramite tre questionari (iniziale Q1, intermedio Q2 e finale Q3) e le considerazioni emerse durante le lezioni e gli interventi didattici mirati a lavorare sugli atteggiamenti, che sono stati audio-registrati o annotati.

\begin{tabular}{|c|c|c|}
\hline Fasi & Periodi & Strumenti \\
\hline $\begin{array}{l}\text { Prima } \\
\text { Raccolta dei dati in entrata e analisi della situazione } \\
\text { iniziale. }\end{array}$ & Settembre 2017 & $\begin{array}{l}\text { Q1: Questionario iniziale } \\
\text { (Allegato 1) }\end{array}$ \\
\hline $\begin{array}{l}\text { Seconda } \\
\text { Interventi nei moduli della formazione relativi al tema } \\
\text { degli atteggiamenti e basati sulla condivisione, } \\
\text { discussione e scambio. } \\
\text { Progettazione e implementazione di interventi didattici } \\
\text { mirati a promuovere un atteggiamento positivo verso } \\
\text { la matematica e verso il suo insegnamento. } \\
\text { Monitoraggio dell'evoluzione degli atteggiamenti con } \\
\text { raccolta di dati intermedi. }\end{array}$ & $\begin{array}{l}\text { Settembre } 2017 \text { - maggio } 2019 \\
11 \text { gennaio } 2018 \\
14 \text { gennaio } 2019 \\
\text { Gennaio } 2019\end{array}$ & $\begin{array}{l}\text { Interventi nei moduli previsti } \\
\text { dalla formazione. } \\
\text { Interventi didattici mirati. } \\
\text { Q2: Questionario intermedio } \\
\text { (descrizione nel par. 6.2.2) }\end{array}$ \\
\hline $\begin{array}{l}\text { Terza } \\
\text { Raccolta dei dati a conclusione del secondo anno di } \\
\text { formazione e analisi della situazione finale. }\end{array}$ & Maggio 2019 - agosto 2020 & $\begin{array}{l}\text { Q3: Questionario finale } \\
\text { (Allegato 2) }\end{array}$ \\
\hline
\end{tabular}

Tabella 3. Fasi, periodi e strumenti del progetto AMI.

Tutti e tre i questionari sono stati somministrati agli studenti attraverso un form online da compilare in forma anonima, in momenti diversi dagli orari delle lezioni; all'inizio dello studio è stato assegnato agli studenti un codice univoco per l'identificazione dei questionari (formato dalle lettere "AMI" e da due cifre numeriche), in modo da poter operare confronti longitudinali fra i dati raccolti nelle diverse fasi. La partecipazione è stata incoraggiata, ma si è svolta su base completamente volontaria: il progetto AMl è stato presentato agli studenti come un progetto di ricerca-azione avente per obiettivo anche quello di supportarli nello sviluppo di un atteggiamento positivo nei confronti della matematica e del suo insegnamento.

\section{6 strumenti}

\subsection{Prima fase}

II questionario iniziale Q1 (Allegato 1) è stato progettato allo scopo di indagare l'atteggiamento iniziale degli studenti verso la matematica e la loro disposizione emozionale verso il suo insegnamento. Per realizzarlo ci si è ispirati al questionario testato da Coppola et al. (2012), integrandolo con una sezione specifica di item scelti per sondare le fonti di autoefficacia in matematica, riadattando quelli 
proposti da Usher e Pajares (2009). Le domande erano suddivise in tre sezioni, ognuna dedicata a una specifica componente dell'atteggiamento.

Più in dettaglio, per quanto riguarda la disposizione emozionale sono state poste delle domande aperte, chiedendo di riportare l'emozione provata nei confronti della matematica e nei confronti del suo insegnamento, di qualificare il proprio rapporto con la disciplina, di motivare le risposte fornite e di raccontare un episodio significativo vissuto in relazione alla matematica come allievi.

Per la rilevazione dell'autoefficacia in matematica è stata utilizzata una batteria di 24 item, secondo il modello descritto nel par. 2.2, con risposta su scala Likert di accordo a 5 passi (da $1=$ totalmente falso a 5 = totalmente vero). Due esempi di item sono «Di solito non ho difficoltà a risolvere problemi di matematica» o «Le persone mi dicono che sono portato per la matematica».

Per la dimensione di visione della matematica, è stato chiesto agli studenti di indicare, argomentando, una caratteristica positiva e una negativa della matematica.

\subsection{Seconda fase}

Dopo aver analizzato la situazione iniziale, si è cercato di favorire lo sviluppo di un atteggiamento positivo sia durante i corsi regolari sia con interventi progettati ad hoc, cercando di lavorare sulle tre dimensioni del modello di atteggiamento assunto - disposizione emozionale, senso di autoefficacia, visione - proponendo attività e occasioni di riflessione focalizzate anche solo su una delle tre dimensioni, ma senza perdere di vista la stretta relazione tra esse. Di seguito vengono descritti gli interventi realizzati, distinti tra quelli continuativi abitualmente svolti durante i corsi e i seminari dei moduli regolari e quelli specifici realizzati ad hoc per il progetto.

\subsubsection{Interventi continuativi nei corsi e nei seminari regolari}

Tra gli autori di questo articolo, due ricercatrici sono state docenti di alcuni corsi offerti al primo e al secondo anno della formazione Bachelor, in particolare il corso teorico di Geometria, il corso teorico di Numeri e calcolo / ciclo e il seminario di Numeri e calcolo Il ciclo (si veda la Tabella 2).

All'interno di tali moduli, sono state dichiarate fin dall'inizio le finalità principali della formazione:

- cercare di far riappacificare con la matematica coloro che non avevano avuto un buon rapporto e di consolidare o ampliare il rapporto positivo già instaurato da altri;

- accrescere il senso di autoefficacia degli studenti sia in matematica sia nell'insegnamento della matematica;

- identificare e far evolvere convinzioni che relegassero la matematica a una disciplina arida e fatta unicamente di regole, di giusto o sbagliato, senza spazio per la creatività e la scoperta.

Condividere le proprie emozioni. Per lavorare in quest'ottica, si è fatto spesso leva su come gli studenti si sentivano e su come cercavano di gestire le loro emozioni quando nei corsi teorici dovevano riaffrontare saperi matematici di base e negli incontri dei seminari dovevano progettare una lezione di matematica o l'avevano appena proposta in classe. Un esempio paradigmatico può essere rappresentato dal seminario di rientro dalla pratica blocco del quarto semestre, in cui tutto il bilancio dell'esperienza è stato giocato sulle emozioni provate: una selezione di immagini evocative sono state appese alla lavagna e gli studenti dovevano scrivere il proprio nome accanto a quella che rispecchiava maggiormente il proprio percorso. In seguito, immagine per immagine, ognuno di loro è stato invitato, se lo desiderava, a condividere il motivo per cui aveva scelto quell'immagine, che cosa lo aveva colpito, in che modo si legava alla sua esperienza, e se si trattava più di un'emozione positiva o negativa per lui/lei.

Confrontarsi con i compagni e rivalutare l'errore. Nei corsi e nei seminari è stato inoltre favorito il 
continuo confronto tra i compagni, sia nelle discussioni aperte in aula, sia assegnando compiti da una settimana all'altra per poi costruire la lezione successiva a partire dalle proposte di soluzioni degli studenti, mostrando diverse strategie possibili, anche errate o parziali, e per stimolare la discussione e il confronto. In questo modo, si è voluto anche creare un clima in cui gli studenti si sentissero liberi di proporre una loro strategia, anche se scorretta, parziale o in contrasto con quella di altri compagni, in modo da favorire l'argomentazione e la ricerca di risposte alle diverse problematiche. Si è cercato così di lavorare sulla paura di sbagliare e del confronto con gli altri, per accrescere il senso di autoefficacia in matematica soprattutto nelle dimensioni di mastery e vicarious experience.

Far emergere e sviluppare le proprie convinzioni. Infine, si è cercato di lavorare in profondità sulle convinzioni degli studenti, così da innescare un cambiamento nel rapporto con la matematica e, più in generale, riflettere sul loro futuro ruolo di docenti di matematica. Per intervenire su visioni negative ed esclusivamente strumentali della matematica, che in alcuni casi portano a vere e proprie misconcezioni in relazione ai diversi nuclei fondanti della matematica oggetto di insegnamento nella scuola elementare, anche i corsi a grande gruppo sono stati impostati come lezioni dialogate, in cui gli allievi sono stati invitati a partecipare alla costruzione dei concetti, a esplorare e provare, a risolvere situazioni e inventarne di nuove, a non aver paura di esplicitare i propri dubbi e le proprie difficoltà. Per riportare alcuni esempi, nel corso teorico Geometria, due lezioni sono state dedicate alle misconcezioni più diffuse e difficili da sradicare sulle quali si è riflettuto insieme e durante un incontro gli studenti hanno personalmente realizzato le definizioni dei quadrilateri e la loro classificazione, riflettendo sulle proprietà necessarie e sufficienti per definire una particolare figura; inoltre, ha contribuito a creare un clima di esplorazione in aula anche il fatto che parte del corso sia stato svolto in modalità flipped classroom, con video da visionare prima della lezione e attività da svolgere in aula in presenza durante la lezione successiva (Sbaragli, Carotenuto \& Castelli, 2017). Nel corso Grandezze e misure, una lezione è stata dedicata a riflettere sulla inesistenza di una relazione tra area e perimetro delle figure piane, basandosi su esempi forniti dagli studenti stessi. Nel corso Numeri e calcolo, diverse lezioni sono state dedicate a riprendere i modelli mentali associati alle quattro operazioni, partendo dall'analisi di quanto gli studenti avevano potuto osservare e raccogliere in classe con i loro allievi. Questo modo di far lezione intende portare gli studenti a vedere la disciplina in modo non nozionistico, ma come qualcosa da costruire insieme. Per questo si è cercato di incentivare gli studenti ad affrontare la matematica in gruppo, sia in presenza sia a distanza, così da confrontare i diversi punti di vista e arricchire il proprio sguardo.

Attraverso tali riflessioni si è cercato di far apprezzare questa disciplina anche agli studenti che la ritenevano troppo complessa, portandoli a vedere la complessità come un catalizzatore piuttosto che un freno alla loro immaginazione. Dedicando inoltre del tempo al confronto di diverse convinzioni e diverse strategie risolutive, si è cercato anche di mettere l'accento sulla dimensione del tempo e su quanto sia importante in matematica lasciarlo agli allievi per consentire loro di porsi domande, ragionare, abbandonare strategie risolutive e intraprenderne altre.

Proporre e vivere esperienze positive con la matematica in contesti informali. In questo senso, un'esperienza importante per il rafforzamento e lo sviluppo di un atteggiamento positivo verso la matematica e il suo insegnamento da parte degli studenti è stata la loro partecipazione attiva al festival "Matematicando: a spasso con la matematica per le strade di Locarno", ${ }^{2}$ tenutosi a maggio 2018 a Locarno. Gli studenti, al loro primo anno di formazione, sono stati coinvolti come animatori progettando laboratori e attività durante l'anno accademico per poi realizzarle nelle tre giornate del festival rivolte ad allievi, famiglie, e più in generale all'intera popolazione, con l'intento di promuovere e

2. https://www.matematicando.supsi.ch/eventi/matematicando-festival-2018/. 
far vivere esperienze positive con la matematica e rinnovare il fascino per questa disciplina. Per gli studenti si è trattata di un'occasione preziosa per sperimentare modi di apprendere e di insegnare matematica, fuori dall'aula scolastica, come disciplina viva ed emozionante.

\title{
6.2.2 Interventi specifici realizzati ad hoc
}

In parallelo al percorso regolare dei moduli di matematica, sono stati organizzati degli incontri specifici per discutere delle emozioni scaturite dalle pratiche in classe o in relazione allo studio di particolari temi. Tali discussioni hanno portano inevitabilmente a toccare anche le altre dimensioni dell'atteggiamento.

Primo incontro. II primo di questi momenti si è svolto a gennaio 2018 ed è stato introdotto con una classica attività proposta da Brousseau (1981) come esempio paradigmatico di attività a-didattica. Gli studenti sono stati divisi in gruppi di 4-5 persone e hanno ricevuto un puzzle come quello riportato in Figura 1, con la seguente consegna:

\begin{abstract}
«Dovete costruire un puzzle più grande, simile a quello che vi è stato consegnato: il segmento che misura $4 \mathrm{~cm}$ nel modello dovrà misurare $7 \mathrm{~cm}$ nella vostra riproduzione. Ciascun componente del gruppo dovrà fare uno o due pezzi del puzzle. Quando ogni componente del gruppo avrà finito il lavoro assegnato, provate a mettere insieme i pezzi e a ricostruire il puzzle ingrandito».
\end{abstract}

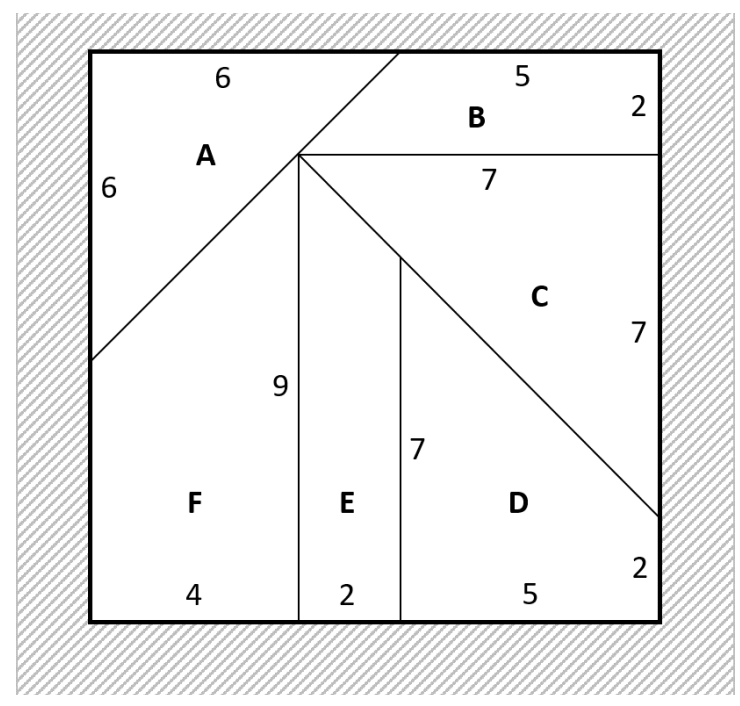

Figura 1. Il puzzle di Brousseau (1981, p. 70)

L'attività mette in gioco aspetti matematici importanti e non banali (a partire dal concetto di similitudine e proporzione) e ingaggia chi la intraprende in una "sfida intellettuale". II fine è univoco: quello di ricostruire un puzzle e, usualmente, questo fa sì che non entrino in gioco aspetti del contratto didattico legati all'interpretazione della richiesta («Cosa vorrà chiedermi il docente con questa domanda?») o della domanda attesa («Cosa vorrà che risponda?»).

La discussione è stata centrata prevalentemente sulle emozioni, con l'obiettivo però di evidenziare un altro aspetto cruciale: il ruolo dell'errore in un'attività di questo tipo. L'errore infatti non è certificato da un'autorità esterna, ma dal non riuscire a ricostruire il puzzle. L'obiettivo della discussione è stato quello di generare negli studenti una curiosità intrinseca (e rara alla fine di un'attività matematica) relativa a quale fosse l'eventuale errore (o gli errori) e quali le cause. L'errore in questa attività non è 
vissuto come un giudizio (negativo) in un certo senso puramente valutativo, ma come un'evidenza che si tramuta in ipotesi di lavoro. La proposta può servire a mostrare come il tipo di attività può creare le condizioni affinché l'errore abbia un carico emozionale legato alla curiosità della scoperta piuttosto che alla paura di sbagliare.

Secondo incontro. Un secondo incontro specifico per parlare delle emozioni provate dagli studenti, della loro origine e del loro eventuale cambiamento si è svolto dopo la somministrazione del questionario intermedio (Q2), costituito dalla seguente domanda aperta: «Che emozione provi ripensando alle attività di matematica che hai proposto nelle tue pratiche in classe? Spiega perché provi questa emozione». Gli studenti hanno avuto una settimana di tempo per rispondere al questionario, poi è seguito un incontro a grande gruppo in aula. All'inizio dell'incontro, gli studenti hanno preso un foglio rosso, verde o giallo a seconda che l'emozione dichiarata nel questionario fosse rispettivamente negativa, positiva, oppure ambivalente. Gli studenti si sono in seguito raggruppati per colore e hanno avviato una discussione sulla base di tre domande stimolo:

- Rispetto all'inizio della formazione, provi le stesse emozioni verso la matematica e verso il fatto che la insegnerai?

- Se sono diverse, in che modo sono cambiate? Secondo te, che cosa le ha fatte cambiare?

- Pensi che i corsi e/o la pratica professionale abbiano avuto un impatto su tali emozioni?

È seguita una discussione aperta in cui liberamente ogni studente ha riportato quanto emerso nei gruppi e ha evidenziato se ci fosse stato un cambiamento nelle emozioni o nel proprio rapporto con la disciplina dall'inizio della formazione e, in caso affermativo, a che cosa fosse dovuto.

\subsection{Terza fase}

Nel questionario finale (Q3, Allegato 2) sono state riprese alcune domande del Q1, per avere una base comune su cui confrontare le risposte, e altre sono state modificate o aggiunte. Nello specifico:

- nella sezione relativa alla disposizione emozionale, è stato nuovamente richiesto di specificare I'emozione provata nei confronti della matematica e del suo insegnamento, motivando la propria risposta, nonché di valutare il proprio rapporto con la disciplina. Diversamente dal Q1, invece, è stato chiesto di raccontare due episodi significativi: uno legato all'esperienza vissuta come studenti del DFA, I'altro vissuto come docenti di matematica durante la pratica professionale;

- nella sezione relativa al senso di autoefficacia, è stata di nuovo proposta la batteria di 24 item sulle fonti di autoefficacia in matematica del Q1, ma chiedendo di riferirsi alla propria esperienza come studenti del DFA. In aggiunta, è stata proposta una batteria di 9 item riadattati dalla ricerca di Zuya et al. (2016) per indagare il senso di autoefficacia come docenti di matematica;

- nella sezione relativa alla visione della matematica e del suo insegnamento, gli studenti dovevano nuovamente indicare una caratteristica positiva e negativa della matematica, argomentando la propria risposta. In più, è stato chiesto quale caratteristica dovesse avere secondo loro un buon docente di matematica.

In questo articolo, faremo riferimento soprattutto all'analisi dei dati in entrata (Q1) e al termine del secondo anno di formazione (Q3), focalizzandoci in particolare sulle dimensioni della disposizione emozionale e dell'autoefficacia, analizzando le mutue relazioni tra queste dimensioni e tenendo conto, quando presenti, di riferimenti espliciti alla visione della disciplina e del suo insegnamento. A differenza del Q1, nel Q3 sono state indagate tutte e tre le dimensioni dell'atteggiamento sia verso la matematica sia verso il suo insegnamento. Ciò comporta che nell'analisi longitudinale si potranno confrontare i dati relativi alla disposizione emozionale nei confronti sia della matematica sia del suo insegnamento e quelli relativi al senso di autoefficacia in matematica; per l'autoefficacia nell'insegnamento della matematica invece si potranno fare delle considerazioni in prospettiva e formulare 
delle ipotesi a partire dai dati raccolti nel Q3. Nell'analizzare questi aspetti, e in particolare la loro eventuale evoluzione nel tempo, ci si servirà anche dell'analisi qualitativa delle motivazioni fornite dagli studenti in riferimento alle emozioni dichiarate.

\section{Analisi dei dati e discussione dei risultati}

Le analisi condotte riguardano la totalità dei questionari raccolti all'inizio della formazione e alla fine del secondo anno.

Degli 80 studenti partecipanti allo studio, 72 hanno risposto al questionario iniziale (Q1) e 46 al questionario finale (Q3); la variazione nella numerosità del campione dipende da diversi fattori, quali ad esempio gli studenti che hanno abbandonato la formazione, che hanno dovuto ripetere il primo anno o che sono subentrati al secondo anno. I due gruppi di studenti che volontariamente hanno risposto al Q1 e al Q3 sono dunque globalmente diversi: alcuni studenti, infatti, hanno risposto al Q1 e non al Q3 e viceversa, e una parte dei rispondenti (37 studenti) al Q1 ha risposto anche al Q3. I due campioni sono dunque non completamente appaiati ma anche non completamente indipendenti. Un confronto puntuale su tutte le risposte non sarebbe quindi corretto per apprezzare la misura del cambiamento, sarà per questo fatto puntualmente solo sui 37 studenti confrontabili. Allo stesso tempo, però, riteniamo utile osservare i dati globali dei due campioni (risposte al Q1 e al Q3), ipotizzando che ogni eventuale differenza di risultati potrebbe essere imputabile a uno o più fattori combinati fra loro, come ad esempio la differenza di età (i rispondenti al Q3 sono più anziani, quindi con un livello di maturazione individuale presumibilmente più elevato), la differenza nelle esperienze professionali compiute (i rispondenti al Q3 hanno potuto svolgere più ore di docenza in aula rispetto ai rispondenti al Q1), o la differenza nel percorso formativo compiuto, in generale e in didattica della matematica ( $\mathrm{i}$ rispondenti al Q3 si trovano al termine del secondo anno della loro formazione come docenti).

I paragrafi seguenti sono dedicati all'analisi delle emozioni e del senso di autoefficacia espressi rispettivamente nei confronti della matematica (par. 7.1) e nei confronti dell'insegnamento della matematica (par. 7.2). Dopo una panoramica su tutte le risposte ricevute a entrambi i questionari, si approfondisce il confronto longitudinale delle risposte dei 37 studenti che hanno partecipato sia al Q1 sia al Q3 (par. 7.1.1 e par. 7.2.1) accompagnato da un'analisi qualitativa delle motivazioni (par. 7.1.2 e par. 7.2.2) che gli studenti hanno fornito a supporto delle proprie emozioni. Queste ultime sono analizzate secondo le tre componenti dell'atteggiamento - disposizione emozionale, autoefficacia, convinzioni - identificate dal modello di Di Martino e Zan (2011), per capire quale di esse abbia inciso maggiormente sull'emozione dichiarata e sul suo eventuale cambiamento.

Disposizione emozionale. Per quanto riguarda la disposizione emozionale, le emozioni dichiarate dagli studenti sia nel Q1 sia nel Q3 vengono classificate rispetto alle due dimensioni definite nei lavori di Pekrun e dei suoi collaboratori (2007; 2017), tradotte da Raccanello e Brondino (2017), e integrate con le spiegazioni che accompagnavano le risposte degli studenti:

- positive o negative, individuando anche quelle ambivalenti (se nella stessa risposta compaiono emozioni sia positive sia negative, ad esempio ansia e speranza) e neutre (nei casi in cui le emozioni provate non siano classificabili né come positive né come negative, anche a seguito della lettura della motivazione fornita dallo studente: un esempio è l'indifferenza);

- attivanti o deattivanti.

Autoefficacia. Per quanto riguarda l'autoefficacia in matematica, le analisi preliminari di affidabilità della scala di misura delle fonti di autoefficacia sono state condotte sui dati raccolti con il Q1 e hanno 
confermato una buona affidabilità dello strumento, mostrando valori di Alpha di Chronbach superiori a 0,70 e indici di correlazione fra gli item con valori da moderati a elevati. Sono state poi condotte analisi descrittive sui questionari raccolti (Q1 e Q3). Inoltre, per confermare l'ipotesi che le differenze osservate fra i due campioni fossero associabili al percorso formativo compiuto in didattica della matematica, è stata condotta un'analisi di confronto fra medie tramite t-test per campioni appaiati sui 37 casi di studenti che hanno risposto a entrambi i questionari.

Per quanto riguarda l'autoefficacia nell'insegnamento della matematica, le analisi di affidabilità della scala di misura dell'autoefficacia nell'insegnamento della matematica sono state condotte sui dati raccolti con il Q3 e hanno confermato una buona affidabilità della scala, con un valore di Alpha di Chronbach uguale a 0,855 . Anche in questo caso sono state condotte analisi descrittive sui questionari raccolti (Q3).

Inoltre, per analizzare il rapporto fra il senso di autoefficacia nell'insegnamento della matematica e le emozioni legate all'apprendimento, è stata condotta un'analisi di confronto fra medie tramite t-test per campioni indipendenti, confrontando i valori medi di autoefficacia nell'insegnamento della matematica fra studenti che hanno riportato un'emozione negativa verso la disciplina e studenti che hanno riportato un'emozione positiva verso la disciplina, basandosi sulle emozioni espresse nel Q3.

\subsection{Risultati relativi alla disposizione emozionale e all'autoefficacia in matematica}

Emozioni verso la matematica. II quadro iniziale delle emozioni dichiarate nei confronti della matematica da parte degli studenti si presenta eterogeneo, con una prevalenza di emozioni negative $(54,2 \%)$, in linea con altri risultati riscontrabili in letteratura (si veda il par. 2). La Tabella 4 mostra la categorizzazione risultante dall'analisi delle 72 risposte raccolte con il Q1.

\begin{tabular}{|l|l|l|l|}
\cline { 2 - 4 } & Frequenza (\%) & Attivanti & Deattivanti \\
\hline Positive & $25(34,7 \%)$ & $\begin{array}{l}\text { 22: piacere, curiosità, speranza, } \\
\text { orgoglio, sorpresa in senso positivo, } \\
\text { sfida3 in senso positivo. }\end{array}$ & 3: appagamento, sollievo, calma. \\
\hline Negative & $39(54,2 \%)$ & $\begin{array}{l}31: \text { ansia, paura, vergogna, } \\
\text { confusione, rabbia, disgusto, } \\
\text { frustrazione. }\end{array}$ & $\begin{array}{l}\text { 8: affaticamento, sconforto, } \\
\text { delusione, noia, tristezza. }\end{array}$ \\
\hline Ambivalenti / & $5(6,9 \%)$ & $\begin{array}{l}\text { 4: piacere/ansia, piacere/vergogna, } \\
\text { sorpresa in senso sia positivo sia } \\
\text { negativo, sfida in senso neutro. }\end{array}$ & 1: indifferenza. \\
\hline Non classificabili ${ }^{4}$ & $3(4,2 \%)$ & Due studenti scrivono «precisione», uno «intrinseco». \\
\hline
\end{tabular}

Tabella 4. Emozioni degli studenti nei confronti della matematica all'inizio della formazione.

Le emozioni ambivalenti o neutre verso la matematica risultano poche $(6,9 \%)$ rispetto a quelle positive $(34,7 \%)$ o negative $(54,2 \%)$ : gli studenti sembrano esprimere posizioni nette riguardo alla valenza della loro disposizione emozionale verso la disciplina.

Alla fine del secondo anno di formazione, invece, la situazione si presenta più incoraggiante. Nella Tabella 5 sono riportate le categorie di emozioni rilevate dalle 46 risposte raccolte con il Q3. 


\begin{tabular}{|l|l|l|l|}
\hline & Frequenza (\%) & Attivanti & Deattivanti \\
\hline Positive & $27(58,7 \%)$ & $\begin{array}{l}\text { 26: piacere, curiosità, sorpresa, } \\
\text { orgoglio, speranza, sfida. }\end{array}$ & 1 : calma. \\
\hline Negative & $16(34,8 \%)$ & $\begin{array}{l}13: \text { ansia, paura, vergogna, } \\
\text { frustrazione. }\end{array}$ & 3: affaticamento, noia. \\
\hline $\begin{array}{l}\text { Ambivalenti / } \\
\text { Neutre }\end{array}$ & $1(2,2 \%)$ & $1:$ piacere/sconforto. & 0. \\
\hline Non classificabili ${ }^{4}$ & $2(4,3 \%)$ & Due studenti scrivono «calcoli» e «praticità». \\
\hline
\end{tabular}

Tabella 5. Emozioni degli studenti verso la matematica alla fine del secondo anno di formazione.

Contrariamente a quanto è emerso dal primo questionario, questa volta sono più le emozioni positive $(58,7 \%)$ rispetto a quelle negative $(34,8 \%)$ e si registra un'unica emozione ambivalente.

In termini di attivazione, le emozioni sia positive sia negative verso la matematica risultano perlopiù attivanti (nell'83\% dei casi nel Q1 e nel 91\% dei casi nel Q3), restituendo un quadro complessivamente positivo, considerato che, come rilevato in letteratura, sono le emozioni negative deattivanti a essere le più rischiose in termini di apprendimento, poiché determinano una diminuzione del livello di motivazione (De Beni, Carretti, Moé \& Pazzaglia, 2008).

Autoefficacia in matematica. Per quanto riguarda il senso di autoefficacia, osservando i valori medi calcolati sulle risposte degli studenti riportati in Tabella 6 , si può notare che la social persuasion è la dimensione con il valore medio più basso $(M=2,68)$, seguita dalla mastery experience $(M=2,88)$, entrambi inferiori al valore centrale di 3 ; il valore medio relativo alla dimensione di physiological state è invece il più elevato $(M=3,57),{ }^{5}$ mentre il valore medio della dimensione di vicarious experience coincide con il valore centrale di 3.

\begin{tabular}{|l|l|l|l|} 
& N. & Media & Deviazione standard \\
\hline Mastery experience & 72 & 2.8796 & .93247 \\
\hline Vicarious experience & 70 & 3.0286 & .83308 \\
\hline Social persuasion & 72 & 2.6782 & 1.00052 \\
\hline Physiological state & 72 & 3.5667 & .96765 \\
\hline
\end{tabular}

Tabella 6. Statistiche descrittive per le fonti di autoefficacia misurate al Q1.

All'inizio della formazione i valori di autoefficacia in matematica non sono dunque elevati, ad eccezione del valore legato all'attivazione fisiologica, e mostrano margini di miglioramento.

Al termine del secondo anno di formazione, come si può osservare in Tabella 7, il valore medio più

5. Valori elevati di questa dimensione indicano l'assenza di stati psicofisiologici negativi associati alla matematica (stress, tensione), essendo gli item formulati in modalità invertita. 
elevato è quello relativo alla mastery experience $(M=3,75)$, seguito da quello di physiological state $(M=3,24)$. Ad eccezione del valore medio di social persuasion $(M=2,96)$, tutti i valori medi sono al di sopra del valore centrale di 3.

\begin{tabular}{|ll|l|l|} 
& N. & Media & Deviazione standard \\
\hline Mastery experience & 43 & 3.7488 & .86310 \\
\hline Vicarious experience & 43 & 3.0271 & .75762 \\
\hline Social persuasion & 43 & 2.9612 & .85127 \\
\hline Physiological state & 43 & 3.2403 & .77006 \\
\hline
\end{tabular}

Tabella 7. Statistiche descrittive per le fonti di autoefficacia misurate al Q3.

Rispetto a quanto rilevato nel Q1, si osserva una differenza per i valori di mastery experience e di social persuasion, che risultano più elevati per il gruppo di rispondenti al Q3. Dal mero confronto dei valori riportati dai rispondenti al Q1 e dai rispondenti al Q3, si è quindi portati ad ipotizzare che sia avvenuto un cambiamento in positivo anche per quanto riguarda i valori di autoefficacia in matematica, ipotesi da confermare con l'analisi di confronto puntuale fra le risposte dei soggetti al Q1 e le risposte degli stessi al Q3, che sarà oggetto del prossimo paragrafo.

\subsubsection{Disposizione emozionale e autoefficacia in matematica a confronto}

II grafico in Figura 2 mostra l'evoluzione delle emozioni dei 37 studenti che hanno partecipato sia al Q1 sia al Q3.

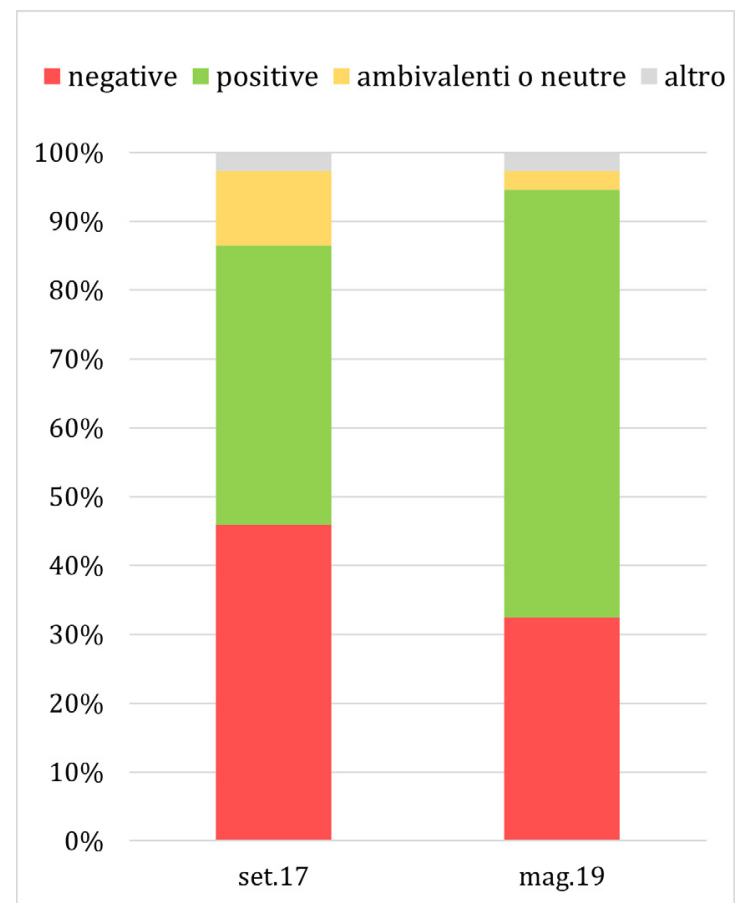

Figura 2. Evoluzione delle emozioni dichiarate dai 37 rispondenti al Q1 e al Q3 verso la matematica. 
Dal confronto longitudinale delle emozioni dichiarate verso la matematica emergono:

- 23 casi di emozioni che cambiano in positivo (9) o restano stabili positive (14);

- 12 casi di emozioni che cambiano in negativo (3) o restano stabili negative (9);

- 2 casi non comparabili, perché in uno dei questionari non è stato possibile classificare l'emozione dichiarata.

L'analisi delle motivazioni fornite dagli studenti (si veda il par. 7.1.2) offre interessanti spunti per interpretare le cause di questa evoluzione.

Per quanto riguarda le dimensioni delle fonti di autoefficacia (Tabella 8), si osserva quanto segue:

- un aumento, sebbene contenuto, dei punteggi in tutte le dimensioni nel tempo (i valori medi misurati nel Q3 sono maggiori rispetto a quelli misurati nel Q1);

- una differenza statisticamente significativa per la dimensione di mastery experience con una differenza media osservata di 0.74 (su una scala da 1 a 5) (t=-5.489, $\mathrm{p}<.001)$.

\begin{tabular}{|ll|l|l|} 
& N. & Media & Deviazione standard \\
\hline Mastery_experience_Q1 & 35 & 2.9905 & .86880 \\
\hline Mastery_experience_Q3 & 35 & 3.7333 & .75861 \\
\hline Vicarious_experience_Q1 & 34 & 3.0196 & .79748 \\
\hline Vicarious_experience_Q3 & 34 & 3.0637 & .80509 \\
\hline Social_persuasion_Q1 & 35 & 2.7714 & 1.04325 \\
\hline Social_persuasion_Q3 & 35 & 3.0571 & .82737 \\
\hline Physiological_state_Q1 & 35 & 3.7714 & .80245 \\
\hline Physiological_state_Q3 & 35 & 3.9476 & .83115 \\
\hline
\end{tabular}

Tabella 8. Numerosità del campione, ${ }^{6}$ media e deviazione standard per ogni dimensione della scala di misura delle fonti di autoefficacia, Q1 e Q3 (dati longitudinali).

Si confermano quindi in parte le differenze osservate nel confronto fra i valori medi riportati dal totale dei rispondenti al Q1 e quelli riportati dal totale dei rispondenti al Q3, ossia un miglioramento nel senso globale di autoefficacia in matematica e in particolare per la dimensione di mastery experience: al termine del secondo anno di formazione, gli studenti si sentono quindi più competenti rispetto ai saperi matematici che saranno chiamati a insegnare.

\subsubsection{Analisi delle motivazioni a supporto delle emozioni verso la matematica}

All'inizio della formazione, le motivazioni con cui i 37 studenti del campione appaiato supportano le emozioni dichiarate verso la matematica richiamano in modo abbastanza omogeneo le tre dimensioni dell'atteggiamento, tutte presenti in misura molto simile sia nel caso di emozioni positive sia nel caso di emozioni negative. La situazione si presenta diversa alla fine del secondo anno della formazione: per le emozioni negative le motivazioni si rifanno primariamente a uno scarso senso di autoefficacia (in 9 casi su 12), mentre per le emozioni positive prevalgono giustificazioni legate alla visione della matematica e del suo insegnamento (in 15 casi su 23).

6. Due dei 37 studenti del campione appaiato non hanno compilato la parte relativa all'autoefficacia in matematica. 
Motivazioni delle emozioni negative. Nelle motivazioni a sostegno delle emozioni negative verso la matematica (17 nel Q1 e 12 nel Q3) si può distinguere la presenza di ciascuna dimensione dell'atteggiamento, come precisato nel seguito.

- Disposizione emozionale. Nel Q1, 7 studenti su 17 rafforzano la connotazione negativa attribuita alla loro disposizione emozionale con motivazioni incentrate su uno scarso interesse, un rapporto negativo o esperienze passate negative. Nello specifico, 2 di questi studenti si riferiscono esplicitamente al proprio docente di scuola media o di scuola media superiore come causa esogena di ansia perché, come scrive AMI32: «ha portato me e la maggior parte della mia classe a provare ansia nei confronti di una materia che se insegnata bene mi è sempre piaciuta» o di frustrazione perché, come afferma AMI69: «mi hanno fatto odiare la materia, in parte perché non la sapevano insegnare in parte perché erano troppo severi». Come vedremo nel seguito, il rapporto di questi due studenti con la matematica è migliorato durante la formazione Bachelor. Nel Q3, 3 studenti su 12 nel motivare l'emozione dichiarata approfondiscono la propria disposizione emozionale negativa, che era già tale nel Q1, riferendosi a un rapporto difficoltoso con la matematica fin dalla scuola media o media superiore, che non è purtroppo migliorato con la formazione. Nel Q3, però, nessuno studente fa più riferimento alla figura dei docenti per motivare emozioni negative.

- Senso di autoefficacia. Nel Q1, 7 studenti su 17 motivano la propria emozione negativa con uno scarso senso di autoefficacia in matematica, come accade per AMI74: «Ansia. Perché non sono mai in grado di dire se quello che sto facendo sia corretto o meno». Nel Q3, questa dimensione dell'atteggiamento risulta prevalente nelle motivazioni a supporto di emozioni negative, presentandosi in 9 casi su 12. Si tratta di studenti che nel Q1 avevano già dichiarato emozioni negative verso la disciplina e che purtroppo non hanno cambiato il proprio rapporto con essa. Di questi studenti, 4 fanno ancora riferimento a sensazioni provate come allievi alla scuola elementare, media o media superiore, come AMI76: «Ansia. Associo questa emozione alla matematica perché mi è capitato spesso di non riuscire a risolvere degli esercizi proposti in classe, come compito o nelle verifiche delle medie o delle superiori. Questo mi procurava molto nervosismo e conseguente ansia per il fallimento». Questo fatto è indicativo perché può significare che tali sensazioni sono così radicate nel vissuto degli studenti che la formazione non è riuscita a scalfirle né ad ammorbidirle. Solo 2 studenti, infine, fanno esplicito riferimento nel Q3 alla formazione ricevuta: entrambi manifestano paura per la matematica per il disagio provato durante gli esami o, come scrive AMI09: «di non essere abbastanza pronto sul lato del sapere docente». È in questo gruppo che si ritrovano i 3 studenti le cui emozioni da positive o ambivalenti si sono trasformate in ansia: la loro autoefficacia in matematica, che probabilmente non si è risollevata durante la formazione, ha prevalso sulla visione della disciplina su cui invece si basavano le emozioni positive o ambivalenti iniziali. Questo può essere il caso, ad esempio, di AMI42 che nel Q1 scrive: «Piacere. Mi piace molto perché si ha un giusto o sbagliato e mi diverto a fare matematica» e nel Q3 scrive: "Ansia. Perché mi sento spesso sotto pressione quando devo risolvere dei calcoli».

- Visione della matematica e del suo insegnamento. Nel Q1, in 4 motivazioni riferite alle 17 emozioni negative si ricavano interessanti informazioni sulla visione che gli studenti hanno della matematica all'inizio del loro percorso di formazione: si tratta prevalentemente di una disciplina percepita come complicata, che pone problemi, e proposta con pochi esempi tangibili. Risulta ricorrente nelle risposte degli studenti la sensazione che la matematica sia in qualche modo "cambiata" negli ultimi anni della scuola media o quando hanno iniziato la scuola media superiore, facendo regredire il loro rapporto con la disciplina; 3 di questi studenti attribuiscono tale "cambiamento" al docente o ai suoi metodi di insegnamento. Nel Q3, 1 sola motivazione delle 12 emozioni negative è legata alla visione della matematica, che risulta strumentale come si evince dalle parole di AMI15: "Ansia. Ogni esercizio ha una soluzione logica, nulla è interpretativo, quindi se non ricordi una determinata formula o concetto teorico o risoluzione non riesci a risolvere il problema». 
Motivazioni delle emozioni positive. Di seguito si precisa, con degli esempi, il riferimento a ciascuna dimensione dell'atteggiamento nelle motivazioni delle emozioni positive verso la matematica (15 nel Q1 e 23 nel Q3).

- Disposizione emozionale. Nel Q1, 6 studenti su 15 rafforzano la loro disposizione emozionale positiva verso la matematica facendo riferimento al fascino della disciplina o a interesse e curiosità personali. È questo il caso degli studenti che dichiarano di provare curiosità verso la matematica, come AMI08: «Ogni volta che vedo qualcosa di nuovo mi incuriosisco. Con la matematica questo rapporto l'ho avuto fin da piccolo e tutt'ora mi incuriosiscono i vari studi, meccanismi, problemi e operazioni». Motivazioni di questo tipo si ritrovano anche nel Q3 in 7 spiegazioni su 23.

- Senso di autoefficacia. Nel Q1, 5 emozioni positive su 15 risultano motivate da un alto senso di autoefficacia in matematica; ciò accade anche nel Q3, seppur con una frequenza più bassa (in 5 casi su 23). Per esempio, è sempre un elevato senso di autoefficacia che porta AMI36 a dichiarare all'inizio della formazione un senso di «Sfida. Perché diventa una sfida capire e risolvere le varie situazioni e problemi» e alla fine del secondo anno di formazione «Piacere. Perché in genere mi riesce bene svolgere questa materia». Solo uno studente, AMI39, al termine del secondo anno di formazione, fa riferimento alla propria autoefficacia come docente: "Calma. Non mi ha mai dato particolari problemi (sia da studente che da docente)».

- Visione della matematica e del suo insegnamento. Nel Q1, in 5 casi su 15, le emozioni positive dichiarate sono legate alla visione della matematica come disciplina ludica e divertente perché «si ha un giusto e sbagliato» (AMI42, piacere) e piacevole perché «senza ordine e regole non si può fare matematica» (AMI63, piacere). Da queste risposte, si deduce che la visione della matematica di questi studenti è di tipo prevalentemente strumentale (nel senso di Skemp, 1976). Anche se ne viene riconosciuta la complessità, la matematica resta una disciplina "complessa ma non impossibile da comprendere» (AMI01, speranza). Nel Q3, gli aspetti legati alla visione predominano nelle motivazioni delle emozioni positive verso la matematica (come anticipato, in 15 casi su 23). Ritroviamo in questo gruppo i 9 studenti che all'inizio della formazione provavano un'emozione negativa (7 casi) o ambivalente (2 casi) verso la matematica; dall'analisi delle motivazioni fornite nel Q3 a sostegno della loro nuova emozione positiva verso la matematica, si può notare che per quasi tutti gli studenti (8 su 9), a causare il cambiamento emozionale, è stata una "nuova" visione della matematica e/o del suo insegnamento, che ne esalta gli aspetti più «ludici» e «divertenti». In particolare, 5 di questi studenti affermano che la loro visione della disciplina è cambiata grazie alla formazione, come accade per AMI32: «Piacere. Dopo aver cominciato il DFA, la mia concezione della matematica è cambiata, infatti soprattutto pensando alla didattica, mi sono avvicinata molto a questa disciplina» e per AMI69: «Curiosità. Perché mi sono accorta che insegnando la matematica scopro sempre più aspetti positivi in questa materia». In relazione a quest'ultimo esempio, può essere interessante osservare che, mentre nel Q1 l'emozione curiosità era motivata facendo appello a tratti distintivi della propria indole personale (fascino e interesse), nel Q3 gli studenti che esprimono curiosità per la matematica si rifanno più alla visione della disciplina, mettendo in evidenza il carattere epistemico dell'emozione provata. Inoltre, da affermazioni come quelle di AMI32 e AMI69, si evince che lo sviluppo di una visione positiva dell'insegnamento della matematica può innescare efficacemente un cambiamento nella visione della matematica, provocando di conseguenza un'evoluzione nelle emozioni provate dagli studenti nei confronti di questa disciplina.

Motivazioni delle emozioni ambivalenti o neutre. Infine, all'origine delle 4 emozioni ambivalenti nel Q1 e dell'unica emozione ambivalente nel Q3, sembra esservi una disposizione emozionale ambivalente. Ne sono un esempio le motivazioni fornite da AMI44 sia nel Q1: «Sorpresa. Scopri di apprezzare alcuni argomenti mentre altri no», sia nel Q3: «Piacere/sconforto. Con la matematica è sempre una scoperta, alcune volte mi evoca sentimenti di tranquillità e gioia, altre volte di sconforto». Questo 
contrasto è dovuto al tipo di compito matematico affrontato, all'argomento trattato o al livello scolastico a cui si fa risalire l'emozione.

\subsection{Risultati relativi alla disposizione emozionale e all'autoefficacia nell'insegnamento della matematica}

Emozioni. All'inizio della formazione, il quadro delle emozioni dichiarate verso il futuro insegnamento della matematica risulta più positivo (Tabella 9 ) rispetto a quello relativo alla disciplina analizzato nel paragrafo precedente.

\begin{tabular}{l|l|l|l|}
\cline { 2 - 4 } & Frequenza (\%) & Attivanti & Deattivanti \\
\hline Positive & $47(65,3 \%)$ & 44: piacere, speranza, curiosità, orgoglio, sorpresa, sfida. & 3: calma. \\
\hline Negative & $5(6,9 \%)$ & 5: ansia, paura. & 0. \\
\hline $\begin{array}{l}\text { Ambivalenti / } \\
\text { Neutre }\end{array}$ & $19(26,4 \%)$ & $\begin{array}{l}\text { 19: piacere/paura, ansia/piacere, paura/sfida, confusione/ } \\
\text { piacere, piacere/affaticamento, ansia/sfida, ansia/calma, } \\
\text { ansia/speranza, confusione/speranza, confusione/sfida, } \\
\text { paura/orgoglio, confusione/piacere; «responsabilità» } \\
\text { (neutra). }\end{array}$ & 0. \\
\hline Non classificabili & $1(1,4 \%)$ & Vuota. & \\
\hline
\end{tabular}

Tabella 9. Emozioni degli studenti verso l'insegnamento della matematica all'inizio della formazione.

Prevalgono le emozioni positive $(65,3 \%)$ mentre le emozioni negative sono poche $(6,9 \%)$, ma sono molte quelle ambivalenti $(26,4 \%)$; cosa che potrebbe essere dovuta al carattere «potenziale» di queste emozioni (Ria \& Chalies, 2003), espresse in riferimento a una situazione immaginata, non ancora vissuta, in cui gli studenti si proiettano e che comunque sembra destare nella maggior parte dei casi un misto tra voglia di mettersi in gioco o di riscattarsi e ansia di riuscire a farcela.

Alla fine del secondo anno di formazione, le emozioni positive sono molte di più $(76,1 \%)$ rispetto a quelle ambivalenti (15,2\%) o a quelle negative (8,7\%). La Tabella 10 raccoglie le categorie di emozioni rilevate dalle 46 risposte ottenute con il Q3.

\begin{tabular}{|l|l|l|l|}
\cline { 2 - 3 } & Frequenza (\%) & Attivanti & Deattivanti \\
\hline Positive & $35(76,1 \%)$ & 29: piacere, curiosità, orgoglio, sfida. & $6:$ calma. \\
\hline Negative & $4(8,7 \%)$ & $4:$ ansia. & 0. \\
\hline $\begin{array}{l}\text { Ambivalenti / } \\
\text { Neutre }\end{array}$ & $7(15,2 \%)$ & $5:$ piacere/ansia, ansia/sfida, paura/sorpresa. & $\begin{array}{l}\text { 2: indifferenza } \\
\text { (neutra). }\end{array}$ \\
\hline
\end{tabular}

Tabella 10. Emozioni degli studenti verso l'insegnamento della matematica alla fine del secondo anno.

Si può quindi ipotizzare che la formazione e la pratica professionale abbiano permesso agli studenti di sviluppare competenze e consapevolezza per contrastare l'ansia rispetto all'insegnamento della matematica.

Anche nei confronti dell'insegnamento della matematica prevalgono le emozioni attivanti (nel $94 \%$ dei casi nel Q1 e nel 83\% dei casi nel Q3) su quelle deattivanti, in particolare lo sono tutte le emozioni 
ambivalenti. Inoltre, si noti che I'unica emozione positiva deattivante dichiarata dagli studenti, sia a settembre 2017 sia a maggio 2019, coincide con la calma. Risulta interessante analizzarne le diverse motivazioni (si veda il par. 7.2.2).

Autoefficacia. Per quanto riguarda il senso di autoefficacia nell'insegnamento della matematica, che è stato misurato solo nel Q3, i valori rilevati sono, in media, per ogni item della scala e per la scala intera, elevati e superiori al valore centrale 3 (Tabella 11). Gli studenti mostrano quindi una "competenza percepita" (di cui si è parlato nel par. 2) nella rappresentazione di sé stessi come futuri docenti di matematica sufficientemente solida.

\begin{tabular}{|l|l|l|l|}
\multicolumn{1}{l|}{} & N & Media & $\begin{array}{l}\text { Deviazione } \\
\text { standard }\end{array}$ \\
\hline Troverò continuamente modi migliori per insegnare la matematica. & 43 & 4.51 & .703 \\
\hline So come insegnare efficacemente i concetti matematici. & 43 & 3.47 & .735 \\
\hline $\begin{array}{l}\text { Capisco i concetti matematici sufficientemente bene da sentirmi } \\
\text { capace di insegnarli a scuola. }\end{array}$ & 43 & 3.84 & .721 \\
\hline $\begin{array}{l}\text { Quando mi troverò ad insegnare la matematica, le domande degli } \\
\text { allievi saranno benvenute. }\end{array}$ & 43 & 4.60 & .660 \\
\hline $\begin{array}{l}\text { Troverò facile utilizzare strumenti per spiegare agli allievi perché la } \\
\text { matematica è utile. }\end{array}$ & 43 & 4.00 & 1.024 \\
\hline Credo di avere le competenze necessarie per insegnare la matematica. & 43 & 3.93 & .704 \\
\hline $\begin{array}{l}\text { Se un allievo ha difficoltà a capire i concetti matematici, credo di } \\
\text { poterlo aiutare a capirli meglio. }\end{array}$ & 43 & 4.05 & .688 \\
\hline $\begin{array}{l}\text { Ho fiducia nel fatto che posso aiutare gli allievi a padroneggiare nuovi } \\
\text { concetti matematici. }\end{array}$ & 43 & 4.23 & .718 \\
\hline $\begin{array}{l}\text { Sento che sarò capace di migliorare i risultati degli allievi in matemati- } \\
\text { ca attraverso una varietà di metodi di insegnamento. }\end{array}$ & 43 & 4.05 & .688 \\
\hline Autoefficacia (intera scala) & 43 & 4.07 & .507 \\
\hline
\end{tabular}

Tabella 11. Numerosità del campione, media e deviazione standard degli item della scala di misura dell'autoefficacia nell'insegnamento della matematica.

\subsubsection{Disposizione emozionale verso l'insegnamento della matematica a confronto}

II grafico in Figura 3 mostra l'evoluzione delle emozioni dichiarate dai 37 studenti che hanno risposto sia al Q1 sia al Q3 nei confronti dell'insegnamento della matematica. 


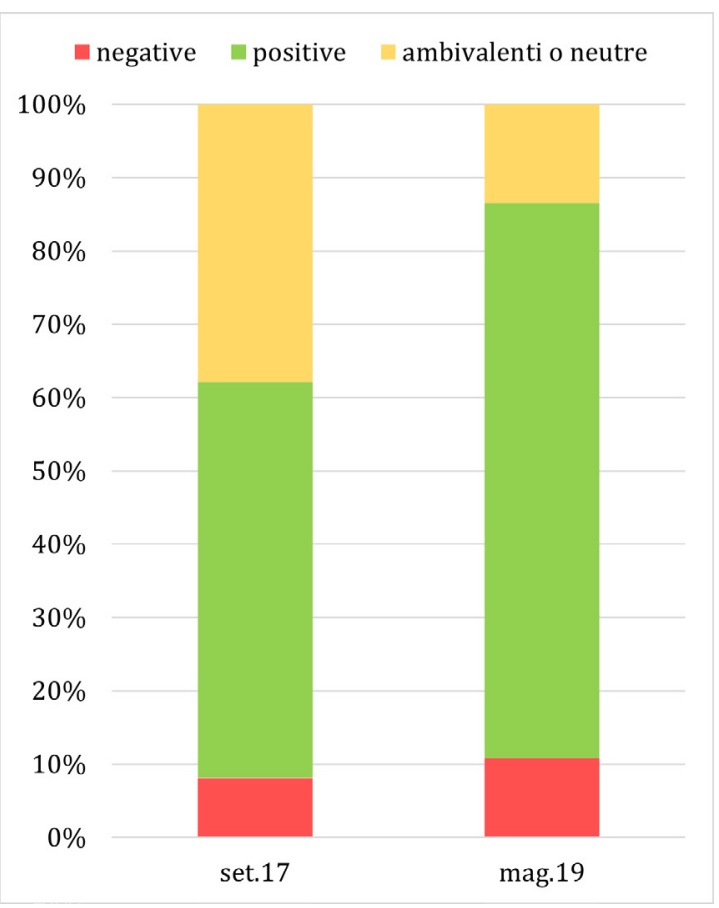

Figura 3. Evoluzione delle emozioni dichiarate dai 37 rispondenti al Q1 e al Q3 verso l'insegnamento della matematica.

Dal confronto longitudinale si riscontrano:

- 29 casi di emozioni che cambiano in positivo (11) o restano stabili positive (18);

- 8 casi di emozioni che cambiano in negativo (4) o restano stabili negative (1) o stabili ambivalenti (3). L'analisi delle motivazioni fornite dagli studenti (si veda il par. 7.2.2) permette di approfondire le possibili ragioni legate a tale evoluzione.

\subsubsection{Analisi delle motivazioni a supporto delle emozioni verso l'insegnamento della matematica} All'inizio della formazione, per motivare le loro emozioni sia positive sia negative gli studenti richiamano e precisano prevalentemente la loro disposizione emozionale verso l'insegnamento della matematica. Inoltre, le motivazioni a supporto delle emozioni ambivalenti si riferiscono in modo importante anche al senso di autoefficacia. Alla fine del secondo anno, aumentano i riferimenti alla visione della matematica e del suo insegnamento soprattutto per le emozioni positive.

Un altro fenomeno interessante che si riscontra nel Q1 è quello della math-redemption (Coppola et al., 2013) presente a inizio formazione in 5 studenti su 37, che dichiarano emozioni negative prevalentemente attivanti verso la matematica - quali ansia, paura, disgusto, sconforto - ma provano emozioni positive (2 studenti) oppure emozioni ambivalenti (3 studenti), tutte attivanti, verso l'insegnamento della matematica. Nonostante il loro vissuto negativo con la disciplina, questi studenti sperano di riconciliarsi con essa grazie alla formazione, come si evince per esempio dalla motivazione data da AMI07 nel Q1: «Sfida. Emozioni contrastanti, quasi di sfida, perché non sono una persona che si scoraggia facilmente. Prenderò questo nuovo percorso come un'opportunità per potermi riappacificare con la materia. Mi piacerebbe poter trovare un nuovo approccio alla materia per potermi divertire». Nell'analisi delle motivazioni presentata in questo paragrafo, risulta particolarmente interessante mostrare come sia evoluta l'emozione di questi 5 studenti nei confronti dell'insegnamento della matematica e, di conseguenza, anche nei confronti della disciplina stessa.

Infine, nelle motivazioni fornite per le emozioni verso l'insegnamento della matematica, gli studenti fanno riferimento sia al proprio atteggiamento sia alle possibili ricadute della propria emozione 
sull'atteggiamento dei loro futuri allievi, mostrando di possedere una buona consapevolezza su questo aspetto e sensibilità professionale. Ciò accade solo in relazione a emozioni attivanti, indipendentemente dalla loro valenza positiva o negativa, e in particolare quando la motivazione fa riferimento alla disposizione emozionale o all'autoefficacia; quando invece gli studenti richiamano la visione della matematica, si tratta sempre della propria visione e non di quella dei bambini.

Motivazioni delle emozioni negative. Delle 3 emozioni negative verso l'insegnamento della matematica registrate nel Q1, una è motivata da un vissuto poco positivo con la materia, mentre 2 sono giustificate da uno scarso senso di autoefficacia, come testimonia AMI61: «Paura. Nel senso che non mi sento capace di spiegare a qualcuno qualche nozione di matematica. Non forse per la complessità della materia ma più per il fatto che è difficile dire se si è capito un argomento o meno, finché poi non devi spiegarlo a qualcuno». Nel Q3, per 2 di questi studenti, I'ansia e la paura si sono trasformate in calma e piacere. Uno di loro invece è rimasto con un'emozione negativa (ansia), aggiungendosi nel Q3 ad altri 3 studenti (si tratta quindi di 4 emozioni negative in tutto per il Q3) che a inizio formazione avevano dichiarato emozioni perlopiù ambivalenti verso l'insegnamento della matematica, con una componente di ansia già presente. Quest'ultima ha purtroppo prevalso sulla componente positiva delle loro emozioni: nel Q3 la loro ansia viene motivata con la paura di sbagliare e di non riuscire a insegnare adeguatamente determinati concetti matematici. Per questi studenti, evidentemente il senso di autoefficacia come futuri docenti di matematica non è ancora sviluppato su basi solide.

Motivazioni delle emozioni positive. Di seguito si precisa ed esemplifica come ciascuna dimensione dell'atteggiamento sia richiamata nelle motivazioni delle emozioni positive degli studenti verso l'insegnamento della matematica (20 nel Q1 e 28 nel Q3).

- Disposizione emozionale. Nel Q1, nella maggior parte delle motivazioni (in 14 casi su 20) gli studenti approfondiscono il carattere positivo attribuito alla propria disposizione emozionale. Nello specifico, 6 di questi studenti sperano di trasmettere il proprio entusiasmo per la disciplina ai loro futuri allievi. Ciò accade anche nel Q3: tra le motivazioni dei 13 studenti sui 28 che fanno appello alla loro disposizione emozionale, 4 si riferiscono alle ricadute sulla disposizione emozionale degli allievi, come AMI76: «Piacere. Provo un'emozione positiva proprio perché voglio far appassionare tutti gli allievi a questa materia proponendo attività per loro entusiasmanti che non provochino ansia e nervosismo». Queste parole sono interessanti perché AMI76, e come lei altri 4 studenti, ha provato e prova ancora al termine del secondo anno emozioni negative verso la matematica ancora legate a esperienze vissute alla scuola media e media superiore: anche se il suo rapporto con la matematica è ancora difficoltoso, la formazione sembra essere riuscita ad intervenire sulle sue emozioni verso l'insegnamento della matematica che da ambivalenti nel Q1 sono risultate positive nel Q3. A proposito di riavvicinamento alla disciplina, nei 5 casi di math-redemption individuati con il Q1, le emozioni degli studenti verso l'insegnamento della matematica sono rimaste stabili positive o sono passate dall'ambivalente al positivo, rafforzando il senso di sfida che essi vivono come occasione per riappacificarsi con la materia; parallelamente, le loro emozioni verso la matematica sono passate da negative a positive (sorpresa, speranza e piacere), tranne in un caso in cui è rimasto un senso di frustrazione legato all'autoefficacia in matematica (scrive AMI07: «Perché non sono mai riuscita a comprendere a fondo gli argomenti affrontati»).

- Senso di autoefficacia. Un alto senso di autoefficacia sembra supportare emozioni positive in 4 studenti su 20 nel Q1 e in 7 studenti su 28 nel Q3. A questo proposito, può essere rilevante osservare che nel Q3 aumentano gli studenti che dichiarano calma (I'unica emozione positiva deattivante presente), e lo fanno richiamando prevalentemente (4 studenti su 5) nelle loro motivazioni il senso di autoefficacia nell'insegnamento della matematica. È il caso ad esempio di AMI03 che, facendo riferimento al percorso di formazione, dichiara nel Q3: «Calma. Sono riuscita, tra- 
mite i corsi proposti, ad approcciarmi positivamente alla materia, ritenendomi maggiormente competente». AMI03 fa parte del gruppo di studenti la cui emozione verso l'insegnamento della matematica ha subito un cambiamento in positivo. Se si analizzano le motivazioni di questi studenti nel Q3, si può notare che il senso di autoefficacia in matematica e nell'insegnamento della matematica gioca un ruolo importante in questa evoluzione.

- Visione della matematica e del suo insegnamento. 6 motivazioni su 20 fanno appello alla visione che gli studenti hanno della matematica e del suo insegnamento alla scuola elementare, come una disciplina giocosa, divertente, concreta e al contempo fondamentale per la crescita dei bambini. Ne è un esempio AMI50, che nel Q1 ripone le sue speranze di costruire una visione positiva della matematica nella formazione che sta per iniziare: «Piacere. Sono affascinata ed entusiasta, vista la prima lezione di matematica che ho seguito al DFA. Sembra essere un approccio più giocoso e tangibile. Perché sono sempre stata avvolta dal mistero della matematica e spero di poterlo finalmente svelare attraverso il DFA e i bambini»; le aspettative di AMI50 non sono state deluse e anche nel Q3 fa riferimento a una visione della matematica «ludica e divertente» facendo riferimento anche agli «sviluppi con gli allievi nella pratica professionale». Nel Q3, in analogia con quanto riscontrato per le emozioni verso la matematica, sembra incidere molto (per 11 studenti su 28) la visione della matematica e del suo insegnamento che gli studenti dichiarano di aver maturato o cambiato. Per alcuni studenti un aspetto interessante è il riferimento esplicito ai corsi e ai seminari seguiti e alla pratica professionale svolta al DFA, come per AMI07: «Piacere. Perché in questi due anni di DFA ho scoperto un nuovo lato della matematica, e cioè che può essere compresa anche in maniera più divertente e ludica» e per AMI42: "Curiosità. Perché durante quest'ultimo anno scolastico ho scoperto alcuni aspetti legati al senso della matematica e sono curiosa di scoprirne altri e di poterli presentare anche ai bambini».

Motivazioni delle emozioni ambivalenti o neutre. Segue infine la descrizione, con alcuni esempi, di come ciascuna dimensione dell'atteggiamento sia richiamata dagli studenti nel motivare emozioni ambivalenti o neutre verso l'insegnamento della matematica (14 nel Q1, 5 nel Q3).

- Disposizione emozionale. Nel Q1, in 9 delle 14 motivazioni legate a questo tipo di emozione, gli studenti rafforzano il carattere ambivalente della loro disposizione emozionale intrecciandola con le altre dimensioni dell'atteggiamento. Nel Q3, lo stesso accade per 2 motivazioni su 5, come ad esempio quella di AMI41: "Piacere/ansia. Sarò confrontata con argomenti e tematiche che ancora non ho veramente avuto modo di approfondire e sperimentare personalmente pertanto sono consapevole che dovrò lavorare molto. In ogni caso sono curiosa di apprendere nuove sfumature dell'insegnamento di questa disciplina».

- Senso di autoefficacia. Nel Q1, le emozioni ambivalenti sono riferite al senso di autoefficacia in 9 casi su 14, come per AMI32, che lo richiama a supporto della componente positiva dell'emozione: "Ansia/sfida. Sono un po' agitata perché spero di tornarla ad apprezzare come qualche anno fa. Allo stesso tempo sono determinata e convinta che posso farcela! Perché comunque ci tengo ad essere una brava docente [...]». 4 di questi studenti si riferiscono inoltre allo sviluppo dell'autoefficacia dei loro futuri allievi. Nel Q3, le emozioni ambivalenti diminuiscono e prevale nelle motivazioni uno scarso senso di autoefficacia a supporto della componente negativa dell'emozione, come testimonia AMI63: "Ansia/piacere. Perché nonostante abbia capito i concetti a volte ho paura di sbagliare».

- Visione della matematica e del suo insegnamento. Nel Q1, la visione della matematica e del suo insegnamento prevale in 6 motivazioni su 14, e sembra determinare per 4 studenti la componente positiva dell'emozione; è ad esempio il caso di AMI08: «Piacere/paura. Entusiasta perché penso che la matematica ci circondi e si possano trovare mille modi per insegnare lo stesso procedimento a diversi bambini, quindi il mio raggio d'azione è presso che infinito. Mentre il timore sorge pensando a come coinvolgere tutti gli allievi e permettere loro di apprezzare la matemati- 
ca come faccio io». Nel Q3, ciò accade per 2 motivazioni su 5, di cui una interessante a supporto dell'indifferenza (emozione neutra) verso l'insegnamento della matematica: essa viene motivata con una visione della matematica ristretta per quanto concerne la scuola elementare che viene descritta come una "tipologia" di matematica» (AMI33) fatta di concetti semplici, con cui non si incontrano particolari difficoltà e che, per questo, non spaventa. Queste parole confermano una concezione ricorrente anche in altre spiegazioni, al di là della loro valenza, ossia che la matematica che si insegna e si impara alle elementari sia una "matematica di tipo diverso" rispetto a quella che si apprende alle medie e alle superiori.

\section{Conclusioni}

In questo studio sono stati indagati gli atteggiamenti dei futuri docenti di scuola elementare del Canton Ticino verso la matematica e verso il suo insegnamento, partendo dalle ipotesi che l'autoefficacia in matematica e nell'insegnamento della matematica siano strettamente legate fra loro nonché alle emozioni provate nei confronti della disciplina e del suo insegnamento, e che su tali aspetti sia possibile lavorare grazie a interventi formativi mirati che considerino, oltre ai saperi disciplinari, didattici e pedagogici, anche gli aspetti affettivi legati alla disciplina.

I risultati hanno messo in evidenza che anche nel contesto ticinese, così come avviene nella maggior parte dei Paesi in cui sono state condotte ricerche simili, I'atteggiamento con cui gli studenti iniziano la formazione per diventare insegnanti di scuola elementare risulta perlopiù negativo nei confronti della matematica: le emozioni iniziali degli studenti sono in gran parte negative e il senso di autoefficacia in matematica generalmente non è elevato. La situazione è invece assai diversa quando gli studenti si riferiscono all'idea di insegnare matematica in futuro: solo pochi manifestano in questo caso emozioni negative, mentre la maggior parte prova emozioni positive o ambivalenti.

Questo risultato è incoraggiante, ma da solo potrebbe non essere sufficiente per sostenere gli studenti nel corso della loro formazione: il percorso dovrebbe essere strutturato in modo da far evolvere gli atteggiamenti positivi dei futuri docenti verso la matematica e verso il suo insegnamento e, di conseguenza, il senso di autoefficacia come futuri insegnanti.

A tale scopo, è risultato efficace progettare interventi che mirassero a sviluppare anche solo una delle tre dimensioni dell'atteggiamento per ottenere effetti positivi sulle altre. Dalle analisi, infatti, è emerso che le emozioni, soprattutto quelle negative, sia verso la matematica sia verso il suo insegnamento, sono legate in maggior misura al senso (scarso, nel caso delle emozioni negative) di autoefficacia in matematica. Per questo motivo, soprattutto nei seminari dei moduli regolari, si è cercato di insistere su questa dimensione di "competenza percepita", invitando ad esempio gli studenti a prendersi il merito della riuscita dei percorsi progettati e implementati, del raggiungimento degli obiettivi prefissati, e aiutandoli a individuare i punti di forza e quelli da migliorare nel lavoro svolto. Peraltro, la capacità di analizzare il proprio operato è uno degli aspetti fondamentali del "Profilo delle competenze" 7 previsto per il Bachelor, indica le competenze ritenute essenziali per iniziare la carriera professionale e che orienta l'impostazione della formazione e il percorso dello studente. Nelle discussioni svolte in classe è stata anche data particolare attenzione al mostrare i diversi processi messi in atto dagli studenti stessi: lo sviluppo di una visione di una matematica che valorizza i processi e le argomentazioni parte anche dalla proposta di attività che permettono strade diverse e dall'enfasi che viene data in fase di discussione a tali strade. 
Dal confronto longitudinale sui dati raccolti all'inizio e alla fine della formazione sull'autoefficacia in matematica emergono ulteriori risultati incoraggianti: gli studenti mostrano infatti un accresciuto senso di autoefficacia in matematica, in particolare sulla dimensione di mastery experience, che risulta significativamente più elevata alla fine del secondo anno di formazione. Tali risultati sono da interpretare positivamente sia per il valore che ne deriva per la formazione in sé (che quindi sembra essere stata efficace nello stimolare una accresciuta competenza percepita in matematica), sia perché incoraggiano, di nuovo, ad intervenire laddove si hanno maggiori probabilità di miglioramento, per rafforzare anche altri aspetti (nello specifico, le emozioni e il senso di autoefficacia come docenti). I risultati infatti mostrano un'evoluzione degli atteggiamenti in corso per molti studenti in positivo, ma va anche ricordato che per alcuni studenti tale evoluzione è in negativo; ciò può dipendere dai risultati ottenuti nella formazione o dall'inefficacia della formazione stessa da questo punto di vista. Va infatti tenuto in considerazione che questo progetto ha coinvolto solo alcuni corsi e seminari, e quindi I'azione prevista potrebbe essere approfondita e migliorata. Uno sviluppo futuro di questa ricerca-azione sul piano della formazione potrebbe prevedere di introdurre in tutti i moduli di matematica dei momenti in cui la finalità, in modo ancora più esplicito e strutturato, sia quella di lavorare sull'atteggiamento verso la matematica e il suo insegnamento, in maniera coordinata tra tutti i docenti.

Si sottolinea inoltre che intervenire sul senso di autoefficacia in matematica permette di incidere sul senso di autoefficacia come docenti (e viceversa), e, come dimostrato dalla letteratura, che c'è un legame anche fra l'autoefficacia come docenti e le emozioni verso l'insegnamento della matematica. Le analisi condotte sui dati raccolti hanno infatti consentito di osservare che l'autoefficacia come docenti è diversa negli studenti in funzione dell'emozione associata all'insegnamento della disciplina. Dal confronto condotto sui valori medi di autoefficacia nell'insegnamento della matematica fra studenti che alla fine del percorso di formazione hanno riportato un'emozione negativa o ambivalente in relazione all'insegnamento della matematica, e studenti che hanno riportato un'emozione positiva in relazione all'insegnamento della matematica, è emerso come i primi (studenti con emozioni negative) mostrassero valori mediamente e significativamente $(p<.05)$ più bassi $(M=3,65)$ degli studenti con emozioni positive $(M=4,21)$. Questo test conferma l'esistenza di una relazione significativa fra le disposizioni emozionali verso l'insegnamento della disciplina e la competenza percepita verso sé stessi come futuri insegnanti: per gli studenti che provano emozioni negative verso l'insegnamento della matematica il senso di autoefficacia come docenti è significativamente più basso rispetto agli studenti che provano un'emozione positiva.

Un altro dato interessante che emerge dalla ricerca è che il ruolo della visione della matematica risulta cruciale nelle spiegazioni degli studenti che alla fine del secondo anno di formazione hanno manifestato emozioni positive verso la matematica. II ruolo di questa dimensione è stato confermato dal confronto longitudinale. La visione della matematica sembra essere così uno dei principali elementi su cui la formazione può intervenire con ampi margini di miglioramento per poter ottenere un effetto indiretto anche sulle emozioni e sull'autoefficacia. A questo scopo, come già osservava Kahle (2008) nella sua ricerca sull'autoefficacia, nei corsi di formazione non è sufficiente proporre approcci concettuali che possano essere riprodotti nelle classi, ma è essenziale aiutare prima di tutto gli studenti a fondare su solide argomentazioni concettuali la loro competenza procedurale in matematica e come docenti. Per fare questo, come sembra essere risultato efficace nel progetto AMl e in linea con altre ricerche (ad esempio, Rolka, Rösken \& Liljedahl, 2006), è necessario offrire occasioni di riflessione agli insegnanti in formazione, chiedendo di (ri)costruire regole e formule nella loro dimensione teorica (come teoremi che derivano da altri assunti e che hanno dei "perché"), e di riflettere sulle connessioni tra i diversi concetti ripresi e studiati.

Studi futuri potrebbero approfondire alcuni aspetti toccati da questo progetto. In particolare, sarebbe auspicabile impostare un disegno di ricerca longitudinale che consenta di verificare l'ipotesi che la formazione (generale e specifica) in didattica della matematica abbia un'influenza sul senso di autoefficacia come docenti, ipotesi che il presente studio consente di formulare ma non di confermare. 
Inoltre, potrebbe essere interessante identificare i momenti e i dispositivi di formazione più efficaci di altri nello sviluppo o nel consolidamento di dimensioni specifiche dell'atteggiamento verso la matematica e verso il suo insegnamento. Ulteriori ricerche potrebbero rilevare le emozioni legate alla disciplina in modo differenziato rispetto alle condizioni possibili di apprendimento (riuscita, in situazione, epistemiche ecc.). Infine, si dovrebbe mirare a coinvolgere un numero di soggetti più elevato e garantirne il coinvolgimento fino al termine del progetto, attraverso accorgimenti metodologici come, ad esempio, prevedere la compilazione dei questionari durante l'orario di lezione.

Nonostante la ricerca in oggetto non abbia potuto approfondire tutti questi aspetti, riteniamo che il percorso progettato e implementato sia risultato molto significativo per le diverse figure coinvolte: docenti, ricercatori e studenti, in quanto ha consentito di focalizzare l'attenzione su aspetti, solitamente sottovalutati dalla quotidianità didattica, che consentono di considerare la formazione dei docenti di scuola elementare nella sua ricca complessità.

\section{Ringraziamenti}

Si ringraziano gli studenti del Bachelor in "Insegnamento per il livello elementare" per la loro partecipazione e per il loro coinvolgimento nel progetto di ricerca.

\section{Bibliografia}

Antognazza, D., Di Martino, P., Pellandini, A., \& Sbaragli, S. (2016). The flow of emotions in primary school problem solving. In K. Krainer \& N. Vondrova (Eds.), Proceedings of CERME 9 - Ninth Congress of the European Society for Research in Mathematics Education (pp. 1116-1122). Charles University in Prague, Faculty of Education.

Bandura, A. (1997). Self-efficacy: The exercise of control. New York: Freeman.

Briley, J. S. (2012). The relationships among mathematics teaching efficacy, mathematics self-efficacy, and mathematical beliefs for elementary pre-service teachers. Issues in the undergraduate mathematics preparation of school teachers, 5, 1-13.

Brousseau, G. (1981). Problèmes de didactiques des décimaux. Recherches en Didactique des Mathématiques, 2(1), 37-125.

Buehl, M., Alexander, A., \& Murphy, P. (2002). Beliefs about schooled knowledge: Domain general or domain specific? Contemporary Educational Psychology, 27(3), 415-449.

Bursal, M., \& Paznokas, L. (2006). Mathematics anxiety and preservice elementary teachers' confidence to teach mathematics and science. School Science and Mathematics, 106(4), 173-180.

Calderhead, J. (1996). Teachers: Beliefs and knowledge. In D. C. Berliner \& R. C. Calfee (Eds.), Handbook of educational psychology (pp. 709-725). New York: Simon \& Schuster Macmillan.

Coppola, C., Di Martino, P., Pacelli, T., \& Sabena, C. (2012). Primary teachers' affect: a crucial variable in the teaching of mathematics. Nordic Studies in Mathematics Education, 17(3-4), 101-118.

Coppola, C., Di Martino, P., Mollo, M., Pacelli, T., \& Sabena, C. (2013). Pre-service primary teachers' emotions: the math-redemption phenomenon. In A. M. Lindmeier \& A. Heinze (Eds.), Proceedings of the 37th Conference of the International Group for the Psychology of Mathematics Education (pp. 225-232). Kiel, Germany.

De Beni, R., Carretti, B., Moé, A., \& Pazzaglia, F. (2008). Psicologia della personalità e delle differenze individuali. II Mulino. 
DECS (2015). Piano di studio della scuola dell'obbligo ticinese. Tratto da ScuolaLab: Disponibile in http://www. pianodistudio.ch (consultato il 09.10.2020).

Di Martino, P., \& Sabena, C. (2011). Elementary pre-service teachers' emotions: shadows from the past to the future. In K. Kislenko (Ed.), Current state of research on mathematical beliefs XVI (pp. 89 - 105). Tallinn university.

Di Martino, P., \& Zan, R. (2011). Attitude towards mathematics: A bridge between beliefs and emotions. ZDM, $43(4), 471-482$.

Esterly, E. J. (2003). A multi-method exploration of the Mathematics teaching efficacy and epistemological beliefs of elementary preservice and novice teachers. Unpublished PhD dissertation in the Graduate School of the Ohio State University.

Evans, J. (2000). Adults' mathematical thinking and emotions: a study of numerate practices. London: Routledge Falmer.

Handal, B. (2003). Teachers' mathematical beliefs: A review. The Mathematics Educator, 13(2), 47-57.

Hannula, M. (2002). Attitude towards mathematics: emotions, expectations and values. Educational Studies in Mathematics, 49(1), 25-46.

Hannula, M. S., Liljedahl, P., Kaasila, R., \& Roesken, B. (2007). Researching relief of mathematics anxiety among pre-service elementary school teachers. In J.-H. Woo, H.-C. Lew, K.-S. Park \& D.-Y. Seo (Eds.), Proceedings of the 31st conference of the International Group for the Psychology of Mathematics Education (vol. 1, pp. 153 - 156). Seoul: PME.

Hill, H. C., Blunk, M. L., Charalambous, C. Y., Lewis, J. M., Phelps, G. C., Sleep, L., \& Ball, D. L. (2008). Mathematical knowledge for teaching and the mathematical quality of instruction: An exploratory study. Cognition and instruction, 26(4), 430-511.

Hodgen, J., \& Askew, M. (2011). Emotion, Identity and Teacher Learning: Becoming a Primary Mathematics Teacher. In C. Day \& J. C. K. Lee (Eds.), New understandings of Teacher's Work: Emotions and Educational Change (pp. 165-183). London: Springer.

Kahle, D. K. (2008). How elementary school teachers mathematical self-efficacy and mathematics teaching self-efficacy relate to conceptually and procedurally oriented teaching practices (Doctoral dissertation, The Ohio State University).

McLeod, D. (1992). Research on affect in mathematics education: a reconceptualization. In D. Grouws (Ed.), Handbook of Research on Mathematics Learning and Teaching (pp. 575-596). New York: MacMillan.

Mihalko, J. C. (1978). The answers to the prophets of doom: mathematics teacher education. In D. B. Aichele (Ed.), Mathematics teacher education: critical issues and trends (pp. 36 - 41). Washington: National Education Association.

Op 'T Eynde, P., De Corte, E., \& Verschaffel, L. (2006). Accepting Emotional Complexity: A Socio-Constructivist Perspective on the Role of Emotions in the Mathematics Classroom. Educational Studies in Mathematics, 63(2), 193-207.

Pajares, F., \& Miller, D. (1994). Role of self-efficacy and self-concept beliefs in mathematical problem solving: a path analysis. Journal of Educational Psychology, 86(2), 193-203.

Pekrun, R. (2006). The control-value theory of achievement emotions: Assumptions, corollaries, and implications for educational research and practice. Educational psychology review, 18(4), 315-341.

Pekrun, R., Frenzel, A. C., Goetz, T., \& Perry, R. P. (2007). The control-value theory of achievement emotions: An integrative approach to emotions in education. In P. A. Schutz \& R. Pekrun (Eds.), Emotion in education 
(pp. 13-36). Amsterdam: Academic Press.

Pekrun, R., Vogl, E., Muis, K. R., \& Sinatra, G. M. (2017). Measuring emotions during epistemic activities: the Epistemically-Related Emotion Scales. Cognition and Emotion, 31(6), 1268-1276.

Raccanello, D., \& Brondino, M. (2017). Achievement Emotions Adjective List (AEAL): User's Manual. Unpublished manuscript.

Raymond, A. M. (1996). The development of preservice elementary teachers' beliefs about and knowledge of alternative mathematics assessment. Paper presented at Annual Meeting of the North American Chapter of the International Group for the Psychology of Mathematics Education, Panama City, Florida, 1996.

Ria, L., \& Chalies, S. (2003). Dynamique émotionnelle et activité: le cas des enseignants débutants. Recherche et formation, 42, 7-19.

Rolka, K., Rösken, B., \& Liljedahl, P. (2006). Challenging the mathematical beliefs of preservice elementary school teachers. Proceedings of the 30th Conference of the International Group for the Psychology of Mathematics Education, 4, 441-448.

Rowe, A. D., \& Fitness, J. (2018). Understanding the role of negative emotions in adult learning and achievement: A social functional perspective. Behavioral sciences, 8(2), 27.

Russell, J. A. (1980). A circumplex model of affect. Journal of personality and social psychology, 39(6), 11611178.

Sbaragli, S. (2006). Primary School Teachers' beliefs and change of beliefs on Mathematical Infinity. Mediterranean Journal for Research in Mathematics Education, 5(2), 49-76.

Sbaragli, S., Carotenuto, G., \& Castelli, L. (2017). Flipped classroom come approccio per lo sviluppo di competenze (FliSCo). Rapporto interdipartimentale dell'Asse 8. Scuola universitaria professionale della Svizzera italiana, Locarno.

Schoenfeld, A. H. (1983). Beyond the purely cognitive: Beliefs system, social cognition, and metacognition as driving forces in intellectual performance. Cognitive Science, 7, 329-363.

Schoenfeld, A. H. (1992). Learning to think mathematically: Problem solving, metacognition and sense making in mathematics. In A. D. Grouws (Ed.), Handbook of Research on Mathematics Learning and Teaching (pp. 334-370). New York: MacMillan.

Skemp, R. R. (1976). Relational understanding and instrumental understanding. Mathematics teaching, 77(1), 20-26.

Staub, F. C., \& Stern, E. (2002). The nature of teachers' pedagogical content beliefs matters for students' achievement gains: Quasi-experimental evidence from elementary mathematics. Journal of educational psychology, 94(2), 344-355.

Thompson, A. G. (1992). Teachers' Beliefs and Conceptions: a Synthesis of the Research. In A. D. Grouws (Ed.), Handbook of Research on Mathematics Learning and Teaching (pp. 127-145). New York: Macmillan Publishing Company.

Usher, E. L., \& Pajares, F. (2009). Sources of self-efficacy in mathematics: A validation study. Contemporary Educational Psychology, 34, 89-101.

Wood, E. (1987). Math anxiety and elementary teachers: What does research tell us? For the Learning of Mathematics, 1(1), 8-13.

Zuya, H. E., Kwalat, S. K., \& Attah, B. G. (2016). Pre-Service Teachers' Mathematics Self-Efficacy and Mathematics Teaching Self-Efficacy. Journal of Education and Practice, 7(14), 93-98. 